\title{
SUBGROUPS OF BIANCHI GROUPS AND ARITHMETIC QUOTIENTS OF HYPERBOLIC 3-SPACE
}

\author{
FRITZ GRUNEWALD AND JOACHIM SCHWERMER
}

\begin{abstract}
Let $\mathscr{O}$ be the ring of integers in an imaginary quadratic numberfield. The group $\mathrm{PSL}_{2}(\mathscr{O})$ acts discontinuously on hyperbolic 3-space $H$. If $\Gamma \leq \operatorname{PSL}_{2}(\mathscr{O})$ is a torsionfree subgroup of finite index then the manifold $\Gamma \backslash H$ can be compactified to a manifold $M_{\Gamma}$ so that the inclusion $\Gamma \backslash H \leq M_{\Gamma}$ is a homotopy equivalence. $M_{\Gamma}$ is a compact with boundary. The boundary being a union of finitely many 2 -tori. This paper contains a computer-aided study of subgroups of low index in $\operatorname{PSL}_{2}(\mathscr{O})$ for various $\mathscr{O}$. The explicit description of these subgroups leads to a study of the homeomorphism types of the $M_{\Gamma}$.
\end{abstract}

\section{INTRODUCTION}

The study of the group $\mathrm{PSL}_{2}(\mathbb{C})$ consisting of all fractional linear transformations of the projective plane of points at infinity, with complex coefficients, was one of the major topics of mathematics in the 19th century and played an important role in the development of hyperbolic geometry. Already Poincare [30] observed that $\mathrm{PSL}_{2}(\mathbb{C})$ can be identified with the group of orientation preserving isometries of 3-dimensional hyperbolic space $H$. As in the study of the hyperbolic plane, the importance of finding discrete groups of hyperbolic isometries of $H$ was emphasized. Starting with an example by Picard [29] and pursued by Bianchi $[2,3]$ one particularly interesting class of discrete subgroups of $\mathrm{PSL}_{2}(\mathbb{C})$ was considered. It is constructed in the following arithmetic way: Denote by $\mathscr{O}_{d}$ the ring of integers of an imaginary quadratic number field $k=\mathbb{Q}(\sqrt{ } d)$ with $d \in \mathbb{Z}, d<0$ and squarefree. Then $\mathscr{O}_{d}$ gives rise to a discrete group $\Gamma_{d}:=\mathrm{PSL}_{2}\left(\mathscr{O}_{d}\right)<\mathrm{PSL}_{2}(\mathbb{C})$, consisting of fractional linear transformations with coefficients in $\mathscr{O}_{d}$. Each (torsion-free) subgroup $\Gamma$ of finite index in $\Gamma_{d}$ operates properly discontinuously (and freely) on $H$ and a fundamental domain for this $\Gamma$-action on hyperbolic 3-space is noncompact but of finite volume. As early as 1892 Bianchi $[2,3]$ exhibited fundamental domains for some small values of $d$. This was pursued by Swan [40] who extracted group theoretical presentations for $\Gamma_{d}$ with $d<41$.

From various reasons this class of arithmetically defined discrete groups of hyperbolic motions has gained new vivid interest in recent years. First of all, in topology this construction provides us by considering torsion-free subgroups with a bunch of noncompact hyperbolic 3-manifolds which often seem to have special beauty and which deserve further study because of the role they play in

Received by the editors July 19, 1989 and, in revised form, October 1, 1989.

1980 Mathematics Subject Classification (1985 Revision). Primary 11F06. 
3-manifold theory (cf. [41, 42]). Secondly, there is a lot of arithmetic interest in it, which is mainly motivated by the theory of automorphic forms with respect to such groups and its relations with questions in number theory as special values of $L$-functions or the conjectured correspondence with certain classes of elliptic curves. We refer the interested reader to $[8,13,14,17,18]$. Beside that the Bianchi groups $\Gamma_{d}$ are also of interest in their own group theoretical right.

This paper is devoted to a computer aided analysis of subgroups of small index in $\Gamma_{d}$, in particular, we will deal as examples with subgroups of small index in $\Gamma_{d}$ for $d=-1,-2,-3,-5,-6,-7,-11,-19$. There is a fruitful interaction between geometric-topological, group theoretical and arithmetic questions and methods. Our results in here are far from being complete, but because of the lack of any general theory one may think of this as an attempt to describe and to understand certain phenomena which occur in this study.

Using the group-theoretical algorithms described in $\S 1$, together with a lot of help from M. F. Newman at the Australian National University (Canberra) and from J. Neubüser at the RWTH Aachen we were able to accumulate the following tables. First of all, the paper contains complete lists of representatives for the finitely many $\Gamma_{d}$-conjugacy classes of subgroups $\Gamma$ in $\Gamma_{d}$,

(1) of index $\leq 12$ (resp. = 12) for $d=-1$ (resp. for $d=-2$ ) whose commutator factor group $\Gamma^{a b}$ is torsion-free.

(2) of index 12 for $d=-3$,

(3) (i) of index 3 resp. 6 for $d=-7$,

(ii) of index 12 for $d=-7$ whose commutator factor-group $\Gamma^{a b}$ is torsion-free.

Each representative is given in form of a presentation which is chosen as simple as possible. Some additional information is provided in each case:

$d=-1$ : The corresponding hyperbolic 3-manifolds $\Gamma \backslash H$ are described if $\Gamma$ is torsion-free.

$d=-2$ : Here our information is not complete: The eleven $\Gamma_{-2}$-conjugacy classes fall together to (at most) $6 \mathrm{PSL}_{2}(\mathbb{C})$-conjugacy classes. At least one of them is represented by a group with torsion, for two of them the associated arithmetic quotients could be determined. In two of the remaining cases we have found a set of unipotent generators which suggests that these are groups corresponding to a link complement in a 3-sphere (cf. $\S 5$ for more details).

$d=-3$ : Exactly 2 of the 7 classes in this case are represented by torsion-free groups, which are not $\mathrm{PSL}_{2}(\mathbb{C})$-conjugate. The corresponding 3-manifolds are determined. For all classes $\Gamma^{a b}$ is computed.

$d=-7$ : There are three $\Gamma_{-7}$-conjugacy classes of subgroups of index 3 , each represented by a group with torsion. There are 25 (resp. 27) $\Gamma_{-7}$-conjugacy classes of subgroups of index 6 (resp. index 12 with $\Gamma^{a b}$ torsion-free). In some cases where $\Gamma$ is torsion-free we are able to identify the corresponding 3manifold, in some others we only can say that there is a set of unipotent elements generating the group. Therefore, in particular for (3)(ii) our information is not yet complete (cf. $\S 2$ ). In all cases $\Gamma^{a b}$ is computed.

In each case considered we give in a separate list the numbers of $\Gamma_{d}$-conjugacy classes of subgroups of a fixed index $\leq 12$ in $\Gamma_{d}$ together with some additional information on normalizers. There also exist tables for subgroups in $\Gamma_{-11}, \Gamma_{-5}, \Gamma_{-6}, \Gamma_{-15}, \Gamma_{-19}$ which we did not include in this paper (cf. $\S 7$ ). 
Starting with the examples (cf. [27, 37]) of link complements $S^{3}-L$ of arithmetic type (i.e. which are homeomorphic to $\Gamma \backslash H$ for some $\Gamma<\Gamma_{d}$ ) this search was begun to provide some experimental material and insight in dealing with the subsequent natural question if for each $d$ subgroups of $\operatorname{PSL}_{2}\left(\mathscr{O}_{d}\right)$ can yield hyperbolic structures on link complements or not. (This explains also the restriction ' $\Gamma^{a b}$ torsion-free' in (1) and (3)(ii).) As an answer completing previous work we show in $\S 3$ that this definitively (resp. possibly) happens only in the finitely many cases $d=-1,-2,-3,-7,-11$ (resp. $-5,-6,-15,-19$, $-23,-31,-39,-47,-71)$. The methods used are arithmetical ones.

However, beside the complete classification the experimental material described in here provides some hyperbolic 3-manifolds of special beauty. In addition, it is also of arithmetic interest (cf. $\S 3, \S 8)$ and might also be helpful in analyzing Bianchi groups from the group theoretical point of view. This aspect is mainly dealt with in $\S 1$ where we describe the methods by which the tables above were obtained, lay the basis for their interpretation and prove certain group theoretical results which are special to the lattice of subgroups of finite index in Bianchi groups.

Notation. (1) Sometimes we denote the cyclic group $\mathbb{Z} / m \mathbb{Z}, m \in \mathbf{N}$, by $\mathbb{Z} / m$.

(2) For a group $G, G^{a b}$ denotes the abelianization of $G$, i.e., the quotient of $G$ by its commutator subgroup.

(3) If $U$ is a subgroup of a group $G$ we write $N=N(U, G)$ for the index of the normalizer of $U$ in $G$.

\section{Subgroups of Finite INDEX IN BiANCHI GROUPS, GENERALITIES AND COMPUTER-COMPUTATIONS}

1.1. As in the introduction let $k=\mathbb{Q}(\sqrt{ } d)$, with $d \in \mathbb{Z}, d<0$ and squarefree, be an imaginary number field. Denote by $\mathscr{O}_{d}$ the ring of integers in $k$. As $\mathbb{Z}$-module $\mathscr{O}_{d}$ has a basis of the form

$$
\mathscr{O}_{d}=\mathbb{Z} \oplus \mathbb{Z} \omega \quad \text { where } \omega= \begin{cases}\sqrt{ } d, & d \equiv 2,3 \bmod 4, \\ \frac{1+\sqrt{ } d}{2}, & d \equiv 1 \bmod 4 .\end{cases}
$$

The Bianchi group $\Gamma_{d}$ defined by

$$
\Gamma_{d}:=\operatorname{PSL}_{2}\left(\mathscr{O}_{d}\right)=\mathrm{SL}_{2}\left(\mathscr{O}_{d}\right) /( \pm E)
$$

is a discrete subgroup of $\mathrm{PSL}_{2}(\mathbb{C})$ viewed as the group of orientation preserving isometries of the 3-dimensional hyperbolic space $H$. Hence $\Gamma_{d}$ acts properly discontinuously on $H$. Fundamental domains for this action were analyzed by L. Bianchi $[2,3]$ for small values of $d$. This was pursued by Swan [40] and others $[14,33]$. From the existence of nice fundamental domains we get the following

\subsection{Theorem. The Bianchi group $\Gamma_{d}$ is a finitely presented group.}

In fact Lemma 4.12 of [40] shows that it is in principle possible to find a finite presentation for $\Gamma_{d}$. The following argument also shows that $\Gamma_{d}$ is finitely presented. For any prime ideal $\mathbf{p}$ of $\mathscr{O}_{d}$ with $2,3 \nmid \mathbf{p}$ the full congruence group

$$
\Gamma:=\left\{g \in \Gamma_{d} \mid g \equiv 1 \bmod p\right\}
$$


is torsion-free and of finite index in $\Gamma_{d}$. The space $\Gamma \backslash H$ is homotopy equivalent to a compact manifold with boundary [38]. Hence the fundamental group $\pi_{1}(\Gamma \backslash H)$ is finitely presented. But $\Gamma$ is under the monodromy map isomorphic to $\pi_{1}(\Gamma \backslash H)$. Now $\Gamma_{d}$ is finitely presented because it has a subgroup of finite index which has this property. In fact for many small $|d|$ presentations of $\Gamma_{d}$ have been computed, see $[40,11,14]$. Some of them are exhibited in later chapters of this paper.

As we are interested in the subgroups of finite index in $\Gamma_{d}$ the following assertion is of importance.

1.3. Lemma. Let $G$ be a finitely generated group and $n$ a given natural number. Then there exist only finitely many subgroups of index $n$.

There are only finitely many homomorphisms of $G$ to the symmetric group $S_{n}$, since such a map is determined by its value on the generators of $G$. Let $U$ be a subgroup of index $n$. The action of $G$ on $G / U$ defines a map $G \rightarrow S_{n}$ with transitive image. This operation defines a bijection between the set of subgroups of index $n$ and the set of homomorphisms of $G$ to $S_{n}$ with transitive image.

Starting with a finitely presented group $G$ it is clearly possible to list all homomorphisms of $G$ to $S_{n}$ with transitive image. The stabilizer of 1 in $G$ of such an action of $G$ on $\{1, \ldots, n\}$ is of index $n$ in $G$. It is clear how to compute a list of coset representatives of the stabilizer of 1 . To do this effectively for our groups an implementation at Canberra of a low index subgroup program designed by Lepique and Cannon was used. For a good description of these programs see the article [28] of J. Neubüser and the references therein. This computation was done on a UNIVAC 1980.

Starting from a list of subgroups of index $n$ in $G$ the Reidemeister-Schreier algorithm computes presentations for them. This algorithm is described in [20]. We have used the computer program by G. Havas and modified by Richardson [22]. The computation was done on a DEC KA 10.

Even if the index is small the presentations given by the Reidemeister-Schreier algorithm have many generators and relations. The complexity of the presentations can be reduced by Tietze transformation programs [22]. We have used a program designed by $\mathrm{G}$. Havas.

The same low index subgroup and Reidemeister-Schreier programs were used for the computations done at the Lehrstuhl D für Mathematik (J. Neubüser) at the RWTH Aachen.

Given a finite presentation of a group $G$ it is in principle possible by Gausselimination to compute the structure of the commutator factor-group $G^{a b}$. There are effective methods to do this [21]. At Canberra a computer program derived by G. Havas and S. Sterling to compute these data for our programs has been used.

In the tables at the end of this paper we have listed certain subgroups of low indices in $\Gamma_{d}$. We give a set of generators and relations for each subgroup. The letters $A, B, C, E$ refer to certain generators of $\Gamma_{d}$ described in $\S \S 4-$ 6. Most of the interest lies in the torsion-free subgroups of $\Gamma_{d}$. We also have looked for groups with torsion-free commutator factor-group, motivated by results explained in $\S 3$. The following lemma gives some restriction on the index of such subgroups. 
1.4. Lemma. Let $H \leq G$ be a subgroup of finite index in a given group $G$.

(1) If $H$ is torsion-free then $f_{G} \mid[G: H]$ where $[G: H]$ is the index of $H$ in $G$ and $f_{G}$ the lowest common multiple of the orders of finite subgroups of $G$.

(2) If the commutator factor-group $H^{a b}$ of $H$ is torsion-free and if there is an element of order $m$ in $G^{a b}$ then $m \mid[G: H]$.

$\operatorname{ad}(1)$ : Let $T \leq G$ be a finite subgroup. Since $H$ is torsion-free $T$ acts freely on $G / H$. Hence the order of $T$ divides $[G: H]$.

ad(2): Let $t: G^{a b} \rightarrow H^{a b}$ be the transfer homomorphism (cf. [23]), and let $i: H^{a b} \rightarrow G^{a b}$ be the homomorphism induced by inclusion. We have

$$
i \circ t(g)=g^{[G: H]}
$$

for any $g \in G^{a b}$. If $g$ has finite order it is in the kernel of $t$ and then of $i \circ t$. So the order of $g$ divides $[G: H]$.

To apply our lemma we have to know the finite subgroups of $\Gamma_{d}$.

1.5. The only finite subgroups which occur in $S L_{2}(\mathbb{C})$ are the binary polyhedral groups (cf. e.g. [42, §4.4]). Let $x$ be an element of $S L_{2}\left(\mathscr{O}_{d}\right)<S L_{2}(\mathbb{C})$ of finite order $m$. Then $x$ has eigenvalues $\rho$ and $\bar{\rho}$, where $\rho$ is a primitive $m$ th root of unity. Since $\rho+\bar{\rho}$ is an element of $\mathscr{O}_{d} \cap \mathbf{R}=\mathbb{Z}$ we must have $m=1,2,3,4,6$. Thus the only finite subgroups of $S L_{2}(\mathscr{O})$ which can occur are cyclic of the above orders, the quaternion group, binary tetrahedral group and binary octahedral group. Therefore the possible finite subgroups in $\Gamma_{d}=S L_{2}\left(\mathscr{O}_{d}\right) /( \pm E)$ are the cyclic groups of order two and three, the Klein four group $D_{2}=\mathbb{Z} / 2 \times \mathbb{Z} / 2$, the symmetric group $S_{3}$ and the alternating group $A_{4}$.

There is a method actually to decide which finite subgroups occur in a fixed Bianchi group $\Gamma_{d}$. And one can give a formula for the number of conjugacy classes of each finite subgroup type in $\Gamma_{d}$, which is stated in terms of class numbers of extensions of $\mathbb{Q}(\sqrt{ } d)$ [24]. But for our purposes we only need the following

1.6. Lemma (cf. [37]). (1) $\Gamma_{-1}$ contains all possible types of finite subgroups; $f_{\Gamma_{-1}}=12$,

(2) $\Gamma_{-2}$ contains $\mathbb{Z} / 2, \mathbb{Z} / 3, D_{2}, A_{4}$, but no $S_{3} ; f_{\Gamma_{-2}}=12$,

(3) $\Gamma_{-3}$ contains $\mathbb{Z} / 2, \mathbb{Z} / 3, A_{4}, S_{3}$, but no $D_{2}: f_{\Gamma_{-3}}=12$,

(4) $\Gamma_{-7}$ contains $\mathbb{Z} / 2, \mathbb{Z} / 3, S_{3}$, but no $A_{4}$ and $D_{2} ; f_{\Gamma_{-7}}=6$,

(5) $\Gamma_{-11}$ contains $\mathbb{Z} / 2, \mathbb{Z} / 3, A_{4}$, but no $S_{3}$ and $D_{2} ; f_{\Gamma_{-11}}=12$.

The number of conjugacy classes of finite subgroups of $\Gamma_{d}$ is finite. It is in fact possible to compute a set of representatives for the $\Gamma_{d}$-conjugacy classes of finite subgroups. For small $|d|$ this has been done for example in [11].

1.7. Taking a presentation of $\Gamma_{d}$ and our systems of generators for the subgroups $\Gamma$ in our tables various further informations can be obtained. Using a Todd-Coxeter algorithm (cf. [7]) we can obtain the permutation representation of $\Gamma_{d}$ on $\Gamma_{d} / \Gamma$.

Using the lists of conjugacy classes of finite subgroups in $\Gamma_{d}$, it is then, for example, possible to decide whether the $\Gamma$ in question is torsion-free. This was done as exhibited in the tables.

1.8. Sometimes it is clear from the presentations given in the tables for two subgroups $\Gamma_{1}, \Gamma_{2}$ of $\Gamma_{d}$ that these belong to distinct $\Gamma_{d}$-conjugacy classes but 
are isomorphic as groups. In this situation Mostow's rigidity theorem [27, 31] shows that $\Gamma_{1}$ and $\Gamma_{2}$ are $\operatorname{Iso}(H)$-conjugate. Here $\operatorname{Iso}(H)$ is the group of isometries of hyperbolic space $H$. It contains $\mathrm{PSL}_{2}(\mathbb{C})$ as a subgroup of index 2 .

\section{AN EXAMPLE: The BiANChI GRoUP $\Gamma_{-7}$}

2.1. The group $\Gamma_{-7}=\mathrm{PSL}_{2}\left(\mathscr{O}_{-7}\right)$ is generated by the matrices

$$
A=\left(\begin{array}{ll}
1 & 1 \\
0 & 1
\end{array}\right), \quad B=\left(\begin{array}{cc}
0 & -1 \\
1 & 0
\end{array}\right), \quad C=\left(\begin{array}{cc}
1 & \omega \\
0 & 1
\end{array}\right),
$$

where $\omega=(1+\sqrt{-7}) / 2$. A presentation of $\Gamma_{-7}$ is given by the following relations

(1) $B^{2}=1$,

(2) $(A B)^{3}=1$,

(3) $A C A^{-1} C^{-1}=1$,

(4) $\left(B A C^{-1} B C\right)^{2}=1$,

and one computes $\Gamma_{-7}^{a b}=\mathbb{Z} \oplus \mathbb{Z} / 2$. The integral homology of $\Gamma_{-7}$ is determined in [37].

2.2. Since $S_{3}$ is a subgroup of $\Gamma_{-7}(\mathrm{cf} .1 .4,1.6)$ the index of a torsion-free subgroup $\Gamma$ of $\Gamma_{-7}$ is divisible by 6 . However, there are exactly three conjugacy classes of subgroups of index 3 in $\Gamma_{-7}$. A presentation for a representative of each class is contained in Table 1.

Table 2 contains a complete set of representatives of the $\Gamma_{-7}$-conjugacy classes of index 6 in $\Gamma_{-7}$. There are exactly 25 different $\Gamma_{-7}$-conjugacy classes.

(1) The group $\Gamma_{-7}(6,1)$ is torsion-free, and we have $\Gamma_{-7}(6,1)^{a b} \cong \mathbb{Z}^{3}$. The 3-manifold $\Gamma_{-7}(6,1) \backslash H$ is homeomorphic to the complement in $S^{3}$ of the link $L$ given in Figure (1a). In [41, 6.38] it is shown that the group $\Gamma$ generated by the elements $C, B C B, A^{-1} B C B A$ is of index 6 in $\Gamma_{-7}$ and that $\Gamma \backslash H$ is homeomorphic to $S^{3}-L$. Indeed, in $\Gamma_{-7}$ we have

$$
A^{2}=C B C^{-1} B C^{-1} A^{-1} B C^{-1} B A
$$

and hence we have $\Gamma=\Gamma_{-7}(6,1)$.

One easily checks that the groups $\Gamma_{-7}(6, i), i=1,2,3,4$, are isomorphic and therefore by 1.8 conjugate under Iso $(H)$, so they provide homeomorphic 3-manifolds.

(2) The group $\Gamma_{-7}(6,5)$ is torsion-free, and we have $\Gamma_{-7}(6,5)^{a b} \cong \mathbb{Z}^{2}$. The 3-manifold $\Gamma_{-7}(6,5) \backslash H$ is homeomorphic to the complement in $S^{3}$ of the link $L^{\prime}$ shown as Figure 1(b). It was already observed in [41], resp. [19, $\S 3$, Example 2] that the link complement $S^{3}-L^{\prime}$ has a hyperbolic structure as $\Gamma \backslash H$ where $\Gamma$ is a subgroup of index 6 in $\Gamma_{-7}$. An inspection of the list of conjugacy classes of subgroups of index 6 in $\Gamma_{-7}$ shows then that $\Gamma$ is conjugate $\Gamma_{-7}(6,5)$ or $\Gamma_{-7}(6,6)$.

(3) For the nineteen other classes we observe, that $\Gamma_{-7}(6, j)^{a b}, j=$ $7, \ldots, 25$, has torsion, where the torsion coefficients are 2 and 3 except in the case $\Gamma_{-7}(6,21)$ where also $2^{2}$ occurs. It follows that there are (up to conjugacy) no other subgroups $\Gamma$ of index 6 in $\Gamma_{-7}$ besides $\Gamma_{-7}(6, i), i=1, \ldots, 6$, such that $\Gamma \backslash H$ is homeomorphic to a link complement in $S^{3}$. Moreover, the groups $\Gamma_{-7}(6, h), h=11, \ldots, 25$, are not torsion-free. In each case a torsion element is easily extracted from the data given in Table 2. 
(4) In the remaining four cases we have $\Gamma_{-7}(6, m)^{a b} \cong \mathbb{Z}^{2} \times \mathbb{Z} / 3$ for $m=7$ and 10 resp. $\cong \mathbb{Z}^{2} \times(\mathbb{Z} / 3)^{2}$ for $m=8,9$. We have to leave open the question if these groups are torsion-free and if these groups are generated by unipotent elements.

2.3. Table 3 contains a complete list of representatives of the $\Gamma_{-7}$-conjugacy classes of subgroups $\Gamma$ of index 12 in $\Gamma_{-7}$ with torsion-free factor commutator group $\Gamma^{a b}$. There are (up to conjugacy) 27 subgroups of this type. We see that $\Gamma_{-7}(12, k), k=25, \ldots, 27$ are not torsion-free.

(1) For the groups $\Gamma_{-7}(12, i), i=1, \ldots, 6$, we have $\Gamma_{-7}(12, i)^{a b} \cong \mathbb{Z}^{4}$ and all groups are generated by unipotent elements.

The group $\Gamma_{-7}(12,4)$ is of index 2 in $\Gamma_{-7}(6,1)$ and isomorphic to the fundamental group of the complement of the 4-component link shown in Figure 1(c). This can be seen in the following way: Consider the complement of the 3-component link shown in Figure 1(d). Cut the complement along the disk $D$ spanned into the component labelled 1, turn the disk around by $360^{\circ}$ degrees and glue back again. The resulting link is the link $L$ given in Figure 1(a). The link in 1 (c) covers the link in 1 (d) by a cyclic cover of degree 2 which is induced by a $180^{\circ}$-rotation around the axis labelled 1 in Figure $1(\mathrm{c})$. It is clear from our tables that there is a unique Iso $(H)$-conjugacy class of subgroups of index 12 in $\Gamma_{-7}$ which has a representative which is of index 2 in $\Gamma_{-7}(6,1)$ and has $\mathbb{Z}^{4}$ as factor commutator group. The technique to consider homeomorphisms between link complements which are not induced by homeomorphisms of $S^{3}$ was initiated by Thurston in [41, Chapter 6.7].

(2) For the groups $\Gamma_{-7}(12, i), i=7, \ldots, 19$, we have $\Gamma_{-7}(12, i)^{a b} \cong$ $\mathbb{Z}^{3}$. The groups $\Gamma_{-7}(12, k), k=7, \ldots, 10$, can be generated by unipotent elements.

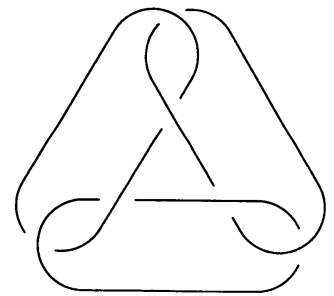

Figure 1(a)

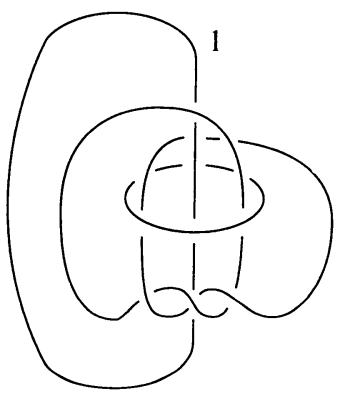

Figure 1(c)

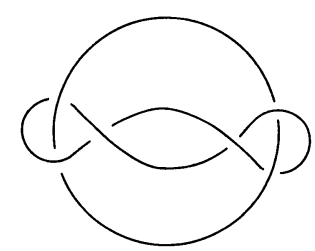

Figure 1(b)

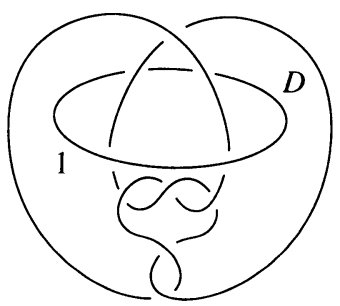

Figure 1(d) 


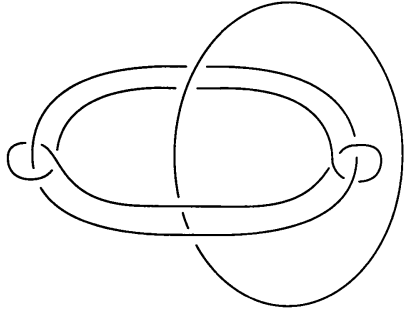

FIGURE 1(e)

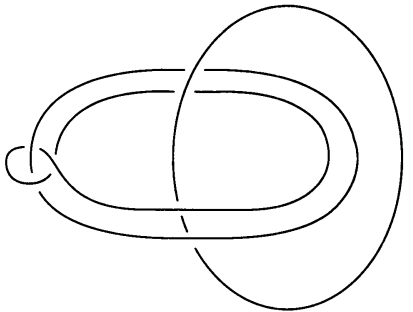

FIGURE 1(f)

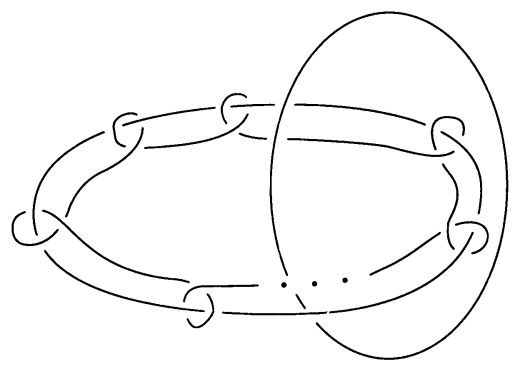

FiguRE 1(g)

The group $\Gamma_{-7}(12,12)$ is isomorphic to the fundamental group of the complement in $S^{3}$ of the 3-component link $M$ shown in Figure 1(e). This can be seen as follows. First of all rearrange the link $L$ (cf. Figure 1(a)) so that it looks like as in Figure 1(f). The link shown in Figure $1(\mathrm{~g})$ covers the link in 1 (f) by a cyclic covering induced by a rotation around the component labelled 1 by $360 / k$ degrees. The special case $k=2$ is shown in Figure 1(e). On the other hand we see from our tables that $\Gamma_{-7}$ contains a unique Iso $(H)$-conjugacy class of subgroups of index 12 which has a representative which is of index 2 in $\Gamma_{-7}(6,1)$ and has $\mathbb{Z}^{3}$ as factor commutator group.

(3) For the groups $\Gamma_{-7}(12, i), i=20, \ldots, 24$, we have $\Gamma_{-7}(12, i)^{a b} \cong \mathbb{Z}^{2}$. Here we do not know if the group can be generated by unipotent elements. The manifolds corresponding to these groups have been studied in [1].

(4) We remark that we also have for the not torsion-free groups $\Gamma_{-7}(12, k)$, $k=25, \ldots, 27$, that $\Gamma_{-7}(12, k)^{a b} \cong \mathbb{Z}^{2}$.

However, we conjecture that some more groups corresponding to link complements are hidden in this list. If the subgroup in question is torsion-free and generated by unipotent elements then Remark 3.2 makes this conjecture plausible. ${ }^{1}$

\footnotetext{
${ }^{1}$ Note added in proof: Many of the open questions in this section have been solved in the recent paper of Fritz Grunewald and Ulrich Hirsch: Link complements arising from arithmetic group actions.
} 
We thank U. Hirsch for his help in working out the examples described in (1) and (2).

2.4. To give an idea how rich the subgroup structure of $\Gamma_{-7}$ is we summarize the numbers of $\Gamma_{-7}$-conjugacy classes of subgroups of index $\leq 12$ in $\Gamma_{-7}$ together with some additional information in the following list; we recall that for a given subgroup $U$ of a group $G$ we write $N=N(U, G)$ for the index of the normalizer of $U$ in $G$.

\begin{tabular}{|ccccccccc|}
\hline Index & Normal & $\begin{array}{c}\text { Self } \\
\text { normalizing }\end{array}$ & $N=2$ & $N=3$ & $N=4$ & $N=5$ & $N=6$ & Total \\
\hline 2 & 3 & & & & & & \\
3 & 1 & 2 & & 2 & & & 3 \\
4 & 3 & 2 & & & 2 & & & 3 \\
5 & 1 & 1 & & & & 1 & & 5 \\
6 & 5 & 6 & 2 & 12 & & & 6 & 25 \\
7 & 1 & 5 & & & & & & 6 \\
8 & 3 & 7 & & & 14 & & & 24 \\
9 & 1 & 24 & & 8 & & & & 33 \\
10 & 3 & 16 & & & & 7 & & 26 \\
11 & 1 & 26 & & & & & & 27 \\
12 & 5 & 95 & 5 & 36 & 5 & & 51 & 197 \\
\hline
\end{tabular}

Numbers of $\Gamma_{-7}$-conjugacy classes of subgroups of index $\leq 12$ in $\Gamma_{-7}$

\section{LINK COMPLEMENTS AND ARITHMETIC QUOTIENTS $\Gamma \backslash H$}

3.1. In the example given in $\S 2$ we have seen that there are subgroups $\Gamma<\Gamma_{-7}$ of finite index such that the associated arithmetic quotient $\Gamma \backslash H$ is homeomorphic to the complement of a link in the 3-sphere. One may ask the natural question for which $d$ examples of this type (i.e. link complements in $S^{3}$ or, more general, in a homology 3-sphere) arise as manifolds $\Gamma \backslash H$, where $\Gamma$ is a subgroup of finite index in $\Gamma_{d}$. A partial answer is given by the following theorem. However, observe that in most cases such an example provides an infinite string of examples by going over to appropriate branched coverings of the link complement started with (cf. [17]).

Theorem. Let $\Gamma_{d}=\operatorname{PSL}_{2}\left(\mathscr{O}_{d}\right), d \in \mathbb{Z}, d<0, d$ squarefree, a Bianchi group. If $d$ is unequal to the numbers contained in the set

$$
\text { B : } \begin{aligned}
\{-1,-2,-3,-5,-6,-7, & -11, \\
& -15,-19,-23,-31,-39,-47,-71\}
\end{aligned}
$$

then for each torsion-free subgroup $\Gamma$ of finite index of $\Gamma_{d}$ the associated hyperbolic 3-manifold $\Gamma \backslash H$ is not homeomorphic to a link complement in a homology 3-sphere. 
Let $\Gamma$ be a subgroup of $\Gamma_{d}$ of finite index; we denote the image of the cohomology with compact supports $H_{c}^{*}(\Gamma \backslash H ; \mathbb{C})$ in $H^{*}(\Gamma \backslash H ; \mathbb{C})$ under the natural map $H_{c}^{*}(\Gamma \backslash H ; \mathbb{C}) \rightarrow H^{*}(\Gamma \backslash H ; \mathbb{C})$ by $H_{!}^{*}(\Gamma \backslash H ; \mathbb{C})$. This cohomology space also has an interpretation as an analytically motivated object in the arithmetic theory of Bianchi groups. More precisely, $H_{1}^{*}(\Gamma \backslash H ; \mathbb{C})$ can be identified with the space $H_{\text {cusp }}^{*}(\Gamma \backslash H ; \mathbb{C})$ of harmonic cuspidal differential forms on the symmetric space $H$ which are invariant under the natural action by $\Gamma$. For this we refer to [17, A4]. This space is also called the cusp cohomology of $\Gamma$ and is closely related to the theory of automorphic forms with respect to $\Gamma$.

If we now assume that $\Gamma \backslash H$ is homeomorphic to a link complement in a homology 3-sphere we have $H_{!}^{*}(\Gamma \backslash H ; \mathbb{C})=\{0\}$ as pointed out in [38]. (Note that the argument given there for $S^{3}-L$ generalizes word by word to the case considered here.) As explained in $[16, \S 1]$ this means from the group theoretical point of view that $\Gamma^{a b}$ is generated by unipotent elements. In turn, the existence of cusp cohomology for the full Bianchi group $\Gamma_{d}$ implies that also $H_{1}^{*}(\Gamma \backslash H ; \mathbb{C}) \neq\{0\}$ for any subgroup $\Gamma$ of $\Gamma_{d}$ (cf. Proof of 1.4. in [16]) and so the quotient $\Gamma \backslash H$ is not homeomorphic to a link complement in a homology 3-sphere.

Now, there are several results pertaining to the existence of cusp cohomology with respect to $\Gamma_{d}$. Starting with Theorem 2.2 in [16] one knows that $H_{!}^{*}\left(\Gamma_{d} \backslash H\right) \neq\{0\}$ for $d$ large enough, more precise, $|d|>5.10^{5}$ (cf. also [17]). In a completely different approach Rohlfs [34] has studied the involution on $\Gamma_{d}$ induced by complex conjugation. This gives rise to an involution $\tau_{!}^{i}$ on $H_{!}^{i}\left(\Gamma_{d} \backslash H ; \mathbb{C}\right)$ resp. $\tau^{i}$ on $H^{i}\left(\Gamma_{d} \backslash H ; \mathbb{C}\right)$ as well. The total Lefschetz number $L\left(\tau, \Gamma_{d}\right)=\sum_{i}(-1)^{i} \operatorname{tr} \tau^{i}$ is related via the fixpoint formula $L\left(\tau, \Gamma_{d}\right)=\chi\left(\left(\Gamma_{d} \backslash H\right)^{\tau}\right)$ to the Euler characteristic of the fixpoint set $\left(\Gamma_{d} \backslash H\right)^{\tau}$ of $\tau$ acting on $\Gamma_{d} \backslash H$. Up to finitely many singular points which arise by torsion elements in $\Gamma_{d}$ the set $\left(\Gamma_{d} \backslash H\right)^{\tau}$ is completely determined in [34] by arithmetic methods. By using the isomorphism $H_{1}^{1}\left(\Gamma_{d} \backslash H ; \mathbb{C}\right) \cong H_{1}^{2}\left(\Gamma_{d} \backslash H ; \mathbb{C}\right)$ and an estimate of the contribution to the total Lefschetz number $L\left(\tau, \Gamma_{d}\right)$ by the cohomology 'at infinity' one gets out of this a lower estimate for $\operatorname{tr} \tau_{!}^{1}$ and so for $\operatorname{dim} H_{1}^{1}\left(\Gamma_{d} \backslash H ; \mathbb{C}\right)$. One gets by this and the results of $\S 3$ in [16] that $H_{1}^{1}\left(\Gamma_{d} \backslash H\right)$ is nontrivial for $d \notin \mathbf{B} \cup\{-14,-87,-111,-119,-159\}$ (cf. 4.4 in [34]). By determining also the singular points alluded to above this approach was completed by Krämer [24], and the numbers $-14,-87,-111,-119,-159$ can be erased from the previous list. In summarized form we have that $H_{!}^{*}(\Gamma \backslash H, \mathbb{C})$ is nontrivial if $d$ is not contained in one of the following sets

$$
\begin{gathered}
S=\{-1,-2,-3,-5,-6,-7,-11,-15,-19\}, \\
V=\{-23,-31,-39,-47,-71\} .
\end{gathered}
$$

By the results of Swan [40] one definitely has that

$$
H_{!}^{*}\left(\Gamma_{d} \backslash H ; \mathbb{C}\right)=\{0\} \text { for } d \in S .
$$

The cases $\Gamma_{d}$ with $d \in V$ were subsequently studied by K. Vogtmann in [43]. Exhibiting an explicit fundamental domain for the action of $\Gamma_{d}$ on a 2-dimensional $\Gamma_{d}$-invariant deformation retract $\Gamma_{d}$ of $H$ (whose construction was given by Mendoza [26], cf. also [37, §3] she showed that also in these cases one has a vanishing result for $H_{\text {cusp }}^{1}\left(\Gamma_{d} \backslash H ; \mathbb{C}\right)$. This result was independently obtained by Krämer [24]. This proves our assertion. 
Remark. Subgroups $\Gamma$ of Bianchi groups give rise to noncompact arithmetic quotients $\Gamma \backslash H$, and nonvanishing results for the cusp cohomology of $\Gamma \backslash H$ obtained out of the theory of automorphic forms have topological implications. For a similar reasoning in the case of a compact arithmetic quotient of $H$ (i.e. the arithmetic group in question is associated to a quaternion algebra) we refer to a recent result of L. Clozel [6].

3.2. In our tables we often see subgroups $\Gamma$ of finite index in a group $\Gamma_{d}$ having the following properties:

(1) $\Gamma$ is torsion-free,

(2) $\Gamma$ is generated by unipotent elements.

The manifold $\Gamma \backslash H$ may as mentioned before be compactified to $\overline{\Gamma \backslash H}$ by adding a finite number of tori. The inclusion $\Gamma \backslash H \rightarrow \overline{\Gamma \backslash H}$ is a homotopy equivalence [38]. Taking a base point in $\Gamma \backslash H$ we get an isomorphism $\Gamma \rightarrow \pi_{1}(\overline{\Gamma \backslash H})$. Filling solid open tori into the boundary we obtain a closed compact manifold $M_{\Gamma}$. From our assumption it is clear that $\pi_{1}(\overline{\Gamma \backslash H})^{a b}$ is generated by the image of $\pi_{1}(\partial(\overline{\Gamma \backslash H}))$. Then it follows that $\pi_{1}\left(M_{\Gamma}\right)^{a b}=1$. Thus $\overline{\Gamma \backslash H}$ is homeomorphic to the complement of a link in the homology 3-sphere $M_{\Gamma}$.

\section{THE BIANCHI GROUP $\Gamma_{-1}$}

4.1. The group $\Gamma_{-1}=\mathrm{PSL}_{2}\left(\mathscr{O}_{-1}\right)$ is generated by the matrices

$$
A=\left(\begin{array}{ll}
1 & 1 \\
0 & 1
\end{array}\right), \quad B=\left(\begin{array}{cc}
0 & -1 \\
1 & 0
\end{array}\right), \quad C=\left(\begin{array}{ll}
1 & i \\
0 & 1
\end{array}\right), \quad E=\left(\begin{array}{cc}
-i & 0 \\
0 & i
\end{array}\right),
$$

where $i=\sqrt{ }-1$. A presentation of $\Gamma_{-1}$ is given by the following relations.

(1) $B^{2}=1$,

(2) $(A B)^{3}=1$,

(3) $A C A^{-1} C^{-1}=1$,

(4) $E^{2}=1$,

(5) $(A E)^{2}=1$,

(6) $(C E)^{2}=1$,

(7) $(B E)^{2}=1$,

(8) $(C B E)^{3}=1$,

and we have $\Gamma_{-1}^{a b}=(\mathbb{Z} / 2)^{2}$. For the homology of $\Gamma_{-1}$ with integral coefficients we refer to [37, Theorem 5.5].

4.2. Since $A_{4}$ is a subgroup of $\Gamma_{-1}$ the index of a torsion-free subgroup $\Gamma$ of $\Gamma_{-1}$ is divisible by 12 (cf. 1.4, 1.6). Table 4 contains a complete set of representatives for the $\Gamma_{-1}$-conjugacy classes of subgroups of index $\leq 12$ in $\Gamma_{-1}$ whose commutator factor group $\Gamma^{a b}$ is torsion-free. We observe that there are $6 \Gamma_{-1}$-conjugacy classes, each having a torsion-free representative and that they all have precisely index 12 . There are subgroups of index $\leq 12$ with torsion in $\Gamma^{a b}$.

For the group $\Gamma_{-1}(12,1)$ we have that the hyperbolic 3-manifold $\Gamma_{-1}(12,1) \backslash H$ is homeomorphic to the complement $S^{3}-W$ of the Whitehead link $W$ in $S^{3}$ (cf. Figure 2a). In fact, the generators given for $\Gamma_{-1}(12,1)$ in Table 4 are the ones used in Example 1 in [44], where this homeomorphism is shown (cf. also [41, 7.17]). The groups $\Gamma_{-1}(12, j), j=2,3,4$, are Iso( $\left.H\right)$ conjugate to $\Gamma_{-1}(12,1)$, so they provide manifolds homeomorphic to $S^{3}-W$.

The commutator factor group of $\Gamma_{-1}(12,5)$ is isomorphic to $\mathbb{Z}^{2}$ and 


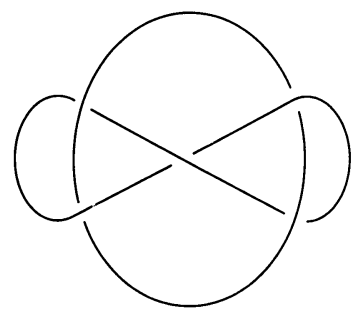

FIGURE 2(a)

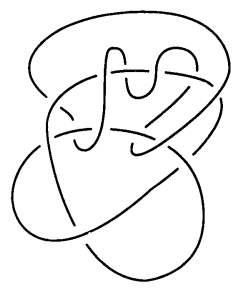

FIGURE 2(b)

$\Gamma_{-1}(12,5) \backslash H$ is homeomorphic to the complement $S^{3}-V$, where $V$ is the link shown in Figure 2(b). This was discovered in [4] where one finds a classification of torsion-free subgroups of index 12 and 24 in $\Gamma_{-1}$. The group $\Gamma_{-1}(12,6)$ is Iso $(H)$-conjugate to $\Gamma_{-1}(12,5)$. Therefore, there are exactly two Iso $(H)$ conjugacy classes of subgroups of index 12 in $\Gamma_{-1}$ with $\Gamma^{a b}$ torsion-free. By [4] these two classes coincide with the $\mathrm{PSL}_{2}(\mathbb{C})$-conjugacy classes of torsion-free subgroups of index 12 in $\Gamma_{-1}$.

In the following list we summarize the numbers of $\Gamma_{-1}$-conjugacy classes of subgroups of index $\leq 12$ in $\Gamma_{-1}$. There is a complete classification of the normal subgroups for indices less than 60 recently given in [10].

\begin{tabular}{|cccccccc|}
\hline Index & Normal & $\begin{array}{c}\text { Self } \\
\text { normalizing }\end{array}$ & $N=3$ & $N=4$ & $N=5$ & $N=6$ & Total \\
\hline 2 & 3 & & & & & & 3 \\
3 & & 1 & 1 & & & & 1 \\
4 & 1 & 3 & & 3 & & & 4 \\
5 & & 4 & & & 4 & & 4 \\
6 & 1 & 6 & 14 & & & 6 & 21 \\
7 & & 2 & & & & & 2 \\
8 & & 7 & & 21 & & & 28 \\
9 & & 10 & & & & & 10 \\
10 & & 7 & & & 20 & & 27 \\
11 & & 6 & & & & & 6 \\
12 & 1 & 33 & 35 & & & 67 & 136 \\
\hline
\end{tabular}

Numbers of $\Gamma_{-1}$-conjugacy classes of subgroups of index $\leq 12$ in $\Gamma_{-1}$.

\section{The Bianchi group $\Gamma_{-2}$}

5.1. The group $\Gamma_{-2}=\operatorname{PSL}_{2}\left(\mathscr{O}_{-2}\right)$ is generated by the matrices

$$
A=\left(\begin{array}{ll}
1 & 1 \\
0 & 1
\end{array}\right), \quad B=\left(\begin{array}{cc}
0 & -1 \\
1 & 0
\end{array}\right), \quad C=\left(\begin{array}{cc}
1 & \omega \\
0 & 1
\end{array}\right),
$$




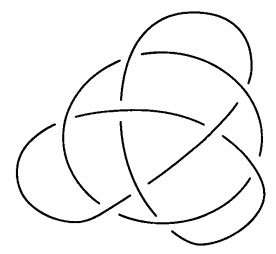

FiguRE 3(a)

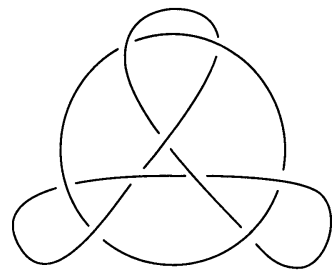

FiguRE 3(b)

where $\omega=\sqrt{ }-2$. A presentation of $\Gamma_{-2}$ is given by the following relations

(1) $B^{2}=1$,

(2) $(A B)^{3}=1$,

(3) $A C A^{-1} C^{-1}=1$,

(4) $\left(B C^{-1} B C\right)^{2}=1$.

As before, we compute $\Gamma_{-2}^{a b}=\mathbb{Z} \times \mathbb{Z} / 6$, and the integral homology of $\Gamma_{-2}$ is determined in [37, Theorem 5.3].

5.2. Since $A_{4}$ is a subgroup of $\Gamma_{-2}$ the index of a torsion-free subgroup of $\Gamma_{-2}$ is divisible by 12 . Out of the subgroups $\Gamma$ of index 12 Table 5 contains a complete list of representatives for the $\Gamma_{-2}$-conjugacy classes whose commutator factor group $\Gamma^{a b}$ is torsion-free. There are (up to $\Gamma_{-2}$-conjugacy) 11 groups $\Gamma_{-2}(12, i), i=1, \ldots, 11$, of this type. We remark that there are no subgroups $\Gamma$ of index 6 in $\Gamma_{-2}$ with $\Gamma^{a b}$ torsion-free.

The group $\Gamma_{-2}(12,1)$ is torsion-free, and we have $\Gamma_{-2}(12,1)^{a b} \cong \mathbb{Z}^{2}$. The arithmetic quotient $\Gamma_{-2}(12,1) \backslash H$ is homeomorphic to the complement in $S^{3}$ of the link $9_{40}^{2}$ (labelled as in [35] and shown in Figure 3(a)). This can be seen in the following way: One knows (cf. [19]) that there are two link complements in $S^{3}$ where the link has two components and which arise as $\Gamma \backslash H$ with $\Gamma<\Gamma_{-2}$ of index 12 . Because of the number of components only the groups $\Gamma_{-2}(12, i), i=1, \ldots, 4$, can provide these manifolds since for these we have $\Gamma_{-2}(12, i)^{a b}=\mathbb{Z}^{2}, i=1, \ldots, 4$. However, $\Gamma_{-2}(12,2)\left(\right.$ resp. $\left.\Gamma_{-2}(12,4)\right)$ is conjugate under Iso $(H)$ to $\Gamma_{-2}(12,1)$ (resp. to $\Gamma_{-2}(12,3)$ ). Using the algorithm in [12] it is possible to compute the Alexander polynomial of $\Gamma_{-2}(12,1)$. This distinguishes the two Iso $(H)$-conjugacy classes and we get our first claim resp. that the arithmetic quotient $\Gamma_{-2}(12,3) \backslash H$ is homeomorphic to the complement in $S^{3}$ of the link $9_{24}^{2}$ shown in Figure 3(b).

For the group $\Gamma_{-2}(12,11)$ we have $\Gamma_{-2}(12,11)^{a b}=\mathbb{Z}^{4}$, and we definitely know that the group contains torsion elements. In the remaining cases $\Gamma_{-2}(12, j), j=5, \ldots, 10$, the question of being torsion-free or not is not decided. However, using 1.8 one can check that $\Gamma_{-2}(12, i)$ and $\Gamma_{-2}(12, i+1)$, $i=5,7,9$, are conjugate under Iso $(H)$. So, there are left three interesting objects. We observe that the groups $\Gamma_{-2}(12,5)$ and $\Gamma_{-2}(12,9)$ are generated by unipotent elements in $\Gamma_{-2}$. Do these groups correspond to link complements in $S^{3}$ ? For the group $\Gamma_{-2}(12,7)$ we do not know if there exists a set of unipotent generators.

5.3. In the following list we summarize the number of $\Gamma_{-2}$-conjugacy classes of subgroups of order $\leq 12$ in $\Gamma_{-2}$ : 


\begin{tabular}{|ccccccccc|}
\hline Index & Normal & $\begin{array}{c}\text { Self } \\
\text { normalizing }\end{array}$ & $N=2$ & $N=3$ & $N=4$ & $N=5$ & $N=6$ & Total \\
\hline 2 & 3 & & & & & & & 3 \\
3 & 4 & 1 & & 1 & & & & 5 \\
4 & 3 & 6 & & & 6 & & & 9 \\
5 & 1 & 2 & & & & 2 & & 3 \\
6 & 13 & 3 & 4 & 27 & & & 3 & 47 \\
7 & 1 & 10 & & & & & & 11 \\
8 & 3 & 20 & & & 30 & & & 53 \\
9 & 4 & 34 & & 4 & & & & 42 \\
10 & 2 & 58 & & & & 10 & & 70 \\
11 & 1 & 62 & & & & & & 63 \\
12 & 16 & 233 & 4 & 96 & 57 & & 120 & 626 \\
\hline
\end{tabular}

6. The Bianchi group $\Gamma_{-3}$

6.1. The group $\Gamma_{-3}=\operatorname{PSL}_{2}\left(\mathscr{O}_{-3}\right)$ is generated by the matrices

$$
A=\left(\begin{array}{ll}
1 & 1 \\
0 & 1
\end{array}\right), \quad B=\left(\begin{array}{cc}
0 & -1 \\
1 & 0
\end{array}\right), \quad C=\left(\begin{array}{cc}
1 & \omega \\
0 & 1
\end{array}\right),
$$

where $\omega=-\frac{1}{2}+\sqrt{ }-3 / 2$. A presentation of $\Gamma_{-3}$ is given by the following relations
(1) $B^{2}=1$,
(2) $(A B)^{3}=1$
(3) $A C A^{-1} C^{-1}=1$,
(4) $\left(A C B C^{-2} B\right)^{2}=1$,
(5) $\left(A C B C^{-1} B\right)^{3}=1$,
(6) $A^{-2} C^{-1} B C B C^{-1} B C^{-1} B C B=1$.

One finds $\Gamma_{-3}^{a b}=\mathbb{Z} / 3$, and the total integral homology of $\Gamma_{-3}$ is determined in [37, Theorem 5.7].

6.2. Since $A_{4}$ is a subgroup of $\Gamma_{-3}$ the index of a torsion-free subgroup of $\Gamma_{-3}$ is divisible by 12 . Now, Table 6 contains a complete list of representatives for the $\Gamma_{-3}$-conjugacy classes of subgroups $\Gamma$ of index 12 in $\Gamma_{-3}$. There are (up to conjugacy) 7 groups of this type. We remark that there are no subgroups $\Gamma$ of index 3 or 6 in $\Gamma_{-3}$ with $\Gamma^{a b}$ torsion-free.

The groups $\Gamma_{-3}(12, i), i=1,2$, are torsion-free and we have $\Gamma_{-3}(12,1)^{a b}$ $\cong \mathbb{Z}$ resp. $\Gamma_{-3}(12,2)^{a b} \cong \mathbb{Z} \times \mathbb{Z} / 5$. The remaining five groups have torsion elements. Therefore, there are exactly two Iso $(H)$-conjugacy classes of torsionfree subgroups of index 12 in $\Gamma_{-3}$. The associated arithmetic quotients can be described as follows: $\Gamma_{-3}(12,1) \backslash H$ is homeomorphic to the complement of the figure-eight knot in $S^{3} .{ }^{2}$ This example is worked out in [32]. As also observed

\footnotetext{
${ }^{2}$ It has recently been proved that the figure-eight knot complement is the only knot complement occurring as a $\Gamma \backslash H$ for an arithmetic group $\Gamma \leq \mathrm{PSL}_{2}(\mathbb{C})$, (A. Reid, Arithmeticity of knot complements).
} 
by Brunner et al. [5, $\S 5$, p. 300] or resp. [45] the group $\Gamma_{-3}(12,2)$ provides as $\Gamma_{-3}(12 / 2) \backslash H$ the manifold obtained by 5 -surgery on one component of the Whitehead link in $S^{3}$ (cf. $\S 4$ ).

6.3. In the following list we summarize the numbers of $\Gamma_{-3}$-conjugacy classes of subgroups of index $\leq 12$ in $\Gamma_{-3}$ :

\begin{tabular}{|ccccccc|}
\hline Index & Normal & $\begin{array}{c}\text { Self } \\
\text { normalizing }\end{array}$ & $N=3$ & $N=4$ & $N=6$ & Total \\
\hline 2 & & & & & & 0 \\
3 & 1 & & & & & 1 \\
4 & & 1 & & 1 & & 1 \\
5 & & 1 & & & & 1 \\
6 & & 1 & 1 & & 1 & 2 \\
7 & & 4 & & & & 4 \\
8 & & 2 & & & & 2 \\
9 & & 1 & & & & 1 \\
10 & & 1 & & & & 1 \\
11 & & & & & & 0 \\
12 & 1 & & & 3 & 3 & 7 \\
\hline
\end{tabular}

7. The Bianchi group $\Gamma_{-5}, \Gamma_{-6}, \Gamma_{-11}, \Gamma_{-15}, \Gamma_{-19}$

7.1. In the case of the group $\Gamma_{-11}$ we have also determined a complete list of representatives for the $\Gamma_{-11}$-conjugacy classes of subgroups of index $\leq 12$ in $\Gamma_{-11}$ whose commutator factor group is torsion-free. There are exactly 55 groups (up to conjugacy) and all are of index 12. Within this list we found, of course, the three known torsion-free subgroups $\Gamma$ in $\Gamma_{-11}$ of index 12 whose associated arithmetic quotient $\Gamma \backslash H$ is homeomorphic to a link complement in $S^{3}$. These are described in Example 3.2 [19], resp. [41]. However, we do not dare to include this huge table into this paper.

7.2. In each of the cases $\Gamma_{d}, d=-5,-6,-15,-19$, the number of conjugacy classes of subgroups of index $\leq 12$ in $\Gamma_{d}$ is very large. Indeed, for $\Gamma_{-5}$ (resp. $\Gamma_{-15}$ ) approximately 35000 (resp. approximately 25000 ) different groups were found. As a remarkable fact in our computations we observed that no group $\Gamma$ could be found whose commutator factor group $\Gamma^{a b}$ is torsion-free. Is this (together with 3.1) a hint that examples of groups $\Gamma$ such that $\Gamma \backslash H$ is homeomorphic to a link complement do not occur in these cases? However, having no hope to get the computation completed the programme was stopped. On the contrary, we have found some subgroups in $\Gamma_{-6}$ with $\Gamma^{a b}$ torsion-free, and having checked ca. 6000 subgroups in $\Gamma_{-19}$ of index $\leq 12$ there is a large number of groups with this property.

\section{A CONCLUDING REMARK ON TORSION ELEMENTS}

As we have seen in $1.5 \Gamma_{d}$ has only elements of finite order 2,3 and 4 . On the other hand, there are torsion-free subgroups of very small index such that the 
abelianized group $\Gamma^{a b}$ has a torsion coefficient $q$ which is different from 2,3 or 4 , i.e., there are torsion elements in the integral homology (or cohomology) of $\Gamma$ which are not detected by the lattice of finite subgroups in $\Gamma$. For example, in Table 6 we have the group $\Gamma_{-3}(12,2)$ of index 12 in $\Gamma_{-3}$ for which one has

$$
\Gamma_{-3}(12,2)^{a b}=\mathbb{Z} \oplus \mathbb{Z} / 5 \mathbb{Z} .
$$

Moreover, we observe in our tables that for a given group $\Gamma$ in there the base elements $p_{i}(\Gamma)$ of the torsion coefficients occurring in

$$
\Gamma^{a b} \cong(\mathbb{Z})^{\mathrm{rank}} \oplus \bigoplus_{i} \mathbb{Z} / p_{i}(\Gamma)^{\nu_{i}} \mathbb{Z}
$$

satisfy the relation

$$
p_{i}(\Gamma) \leq \frac{1}{2}\left[\Gamma_{d}: \Gamma\right] .
$$

It seems to us that this rather strong condition holds in general for a subgroup $\Gamma$ of finite index in $\Gamma_{d}$ and each base element $p_{i}(\Gamma)$ of a torsion coefficient which does not already occur as $p_{i}\left(\Gamma_{d}\right)$ in $\Gamma_{d}^{a b}$. This guess is supported by the same observation in dealing with subgroups of the following type: given an ideal $\mathbf{a} \neq 0$ of $\mathscr{O}_{d}$ we define

$$
\Gamma_{d}(\mathbf{a})_{0}:=\left\{\left(\begin{array}{ll}
a & b \\
c & d
\end{array}\right) \in \Gamma_{d} \mid c \in \mathbf{a}\right\},
$$

and this is obviously a subgroup of finite index in $\Gamma_{d}$. If $\mathbf{a}$ is a prime ideal $\mathbf{p}$ the index $\left[\Gamma_{d}: \Gamma_{d}(\mathbf{p})_{0}\right]$ is given by $N(\mathbf{p})+1$ where $N(\mathbf{p})$ denotes the norm of $\mathbf{p}$. Note that $N(\mathbf{p})=N(\mathbf{q})$ implies that $\Gamma_{d}(\mathbf{p})_{0}$ is isomorphic to $\Gamma_{d}(\mathbf{q})_{0}$. Out of a given presentation for $\Gamma_{d}$ one can find one for $\Gamma_{d}(\mathbf{p})_{0}$. This was used to compute $\Gamma_{d}(\mathbf{p})_{0}^{a b}$ for $d=-1,-2,-3$ and $\mathbf{p}$ a prime ideal of degree one and $N(\mathbf{p}) \leq 400$. For example, checking the table given in $\S 4$ of [9] for $d=-1$ one observes again that (2) is valid. The same holds in the other examples considered (cf. [13]). Some of the torsion elements are related to the arithmetic of algebraic extensions of $\mathbb{Q}(\sqrt{ } d)(\mathrm{cf} .[9, \S 5])$, but in general there is no arithmetic interpretation for them up to now.

\section{ACKNOWLEDGMENT}

The computational work underlying the tables was done in 1980 . The major part of the computer programs were designed and run at the Australian National University in Canberra. We gratefully acknowledge the help of M. F. Newman. We also thank L. S. Sterling and A. W. F. Scheutzow for adapting the existing programs and for running them. A first version of this paper was written in 1983. J.-P. Serre pointed out to us that one of the tables in this first version was incomplete. This mistake occurred in the following way: We had first searched for subgroups of finite index not containing certain torsion elements. Writing up the tables we forgot about this boundary condition. This produced the error mentioned above. Our mistake was restricted to a single table. We eliminated the mistake and then checked our list of subgroups against tables compiled at our request at the RWTH Aachen. We thank J. Neubüser (Lehrstuhl D für Mathematik at the RWTH Aachen) for his help. We also thank Marlies Sieburg for doing the computer work. 


\section{TABLE 1}

All subgroups of index 3 in $\Gamma_{-7}$, up to $\Gamma_{-7}$-conjugacy

\begin{tabular}{|c|c|c|}
\hline$\Gamma_{-7}(3,1)$ & $\begin{array}{l}\text { Generators } \\
g_{1}=C \\
g_{2}=A \\
g_{3}=B C B\end{array}$ & $\begin{array}{l}\text { Relations } \\
\text { (1) } g_{1} g_{2} g_{1}^{-1} g_{2}^{-1}=1 \\
\text { (2) }\left(g_{1} g_{2}^{-1} g_{3}^{-1}\right)^{2}=1 \\
\text { (3) } g_{1} g_{3}^{-1} g_{2}^{-1} g_{3} g_{2} g_{3} g_{1}^{-1} g_{3} g_{2} g_{3}^{-1} g_{2}^{-1} g_{3}^{-1}=1\end{array}$ \\
\hline \multicolumn{3}{|c|}{$\Gamma_{-7}(3,1)^{a b}=\mathbb{Z}^{2} \times \mathbb{Z} / 2 \mathbb{Z}$, not torsion-free, $N=1$} \\
\hline$\Gamma_{-7}(3,2)$ & $\begin{array}{l}g_{1}=C \\
g_{2}=A \\
g_{3}=B A C^{-1} B\end{array}$ & $\begin{array}{l}\text { (1) } g_{1} g_{2} g_{1}^{-1} g_{2}^{-1}=1 \\
\text { (2) }\left(g_{1} g_{3}\right)^{2}=1 \\
\text { (3) } g_{1} g_{2}^{-1} g_{3} g_{2} g_{3} g_{2}^{-1} g_{3}^{-1} g_{2} g_{1}^{-1} g_{3}^{-1} g_{2}^{-1} g_{3}^{-1} g_{2} g_{3}=1\end{array}$ \\
\hline \multicolumn{3}{|c|}{$\Gamma_{-7}(3,2)^{a b}=\mathbb{Z}^{2} \times \mathbb{Z} / 2 \mathbb{Z}$, not torsion-free, $N=1$} \\
\hline$\Gamma_{-7}(3,3)$ & $\begin{array}{l}g_{1}=B \\
g_{2}=A \\
g_{3}=C^{3} \\
g_{4}=C^{-1} B C \\
g_{5}=C B C^{-1}\end{array}$ & $\begin{array}{l}\text { (1) } g_{2} g_{3} g_{2}^{-1} g_{3}^{-1}=1 \\
\text { (2) } g_{1}^{2}=g_{4}^{2}=g_{5}^{2}=1 \\
\text { (3) }\left(g_{2} g_{1}\right)^{3}=\left(g_{2} g_{4}\right)^{3}=\left(g_{2} g_{5}\right)^{3}=1 \\
\text { (4) }\left(g_{1} g_{2} g_{4}\right)^{2}=1 \\
\text { (5) }\left(g_{2} g_{1} g_{5}\right)^{2}=1 \\
\text { (6) }\left(g_{2} g_{5} g_{3} g_{4} g_{3}^{-1}\right)^{2}=1\end{array}$ \\
\hline
\end{tabular}

$\Gamma_{-7}(3,3)^{a b} \cong \mathbb{Z} \times \mathbb{Z} / 2 \mathbb{Z}$, not torsion-free, $N=3$ 
TABLE 2

All subgroups of index 6 in $\Gamma_{-7}$, up to $\Gamma_{-7}$ conjugacy

\begin{tabular}{|c|c|c|}
\hline & Generators & Relations \\
\hline \multirow[t]{2}{*}{$\Gamma_{-7}(6,1)$} & $\begin{array}{l}g_{1}=C \\
g_{2}=A^{2} \\
g_{3}=B C B\end{array}$ & $\begin{array}{l}\text { (1) } g_{1} g_{2} g_{1}^{-1} g_{2}^{-1}=1 \\
\text { (2) } g_{1} g_{2}^{-1} g_{3}^{-1} g_{1} g_{3} g_{1}^{-1} g_{3} g_{2} g_{1}^{-1} g_{3} g_{1}^{-1} g_{3}^{-1} g_{1} g_{3}^{-1}=1\end{array}$ \\
\hline & \multicolumn{2}{|c|}{$\Gamma_{-7}(6,1) \cong \mathbb{Z}^{3}$, torsion-free, link complement group $(2.2), N=3$} \\
\hline \multirow[t]{2}{*}{$\Gamma_{-7}(6,2)$} & $\begin{array}{l}g_{1}=B C A^{-2} B \\
g_{2}=B C^{2} B \\
g_{3}=A\end{array}$ & $\begin{array}{l}\text { (1) } g_{1} g_{2} g_{1}^{-1} g_{2}^{-1}=1 \\
\text { (2) } g_{1} g_{2}^{-1} g_{3}^{-1} g_{1} g_{3} g_{1}^{-1} g_{3} g_{2} g_{1}^{-1} g_{3} g_{1}^{-1} g_{3}^{-1} g_{1} g_{3}^{-1}=1\end{array}$ \\
\hline & \multicolumn{2}{|c|}{ Iso $(H)$-conjugate to $\Gamma_{-7}(6,1), N=3$} \\
\hline \multirow[t]{2}{*}{$\Gamma_{-7}(6,3)$} & $\begin{array}{l}g_{1}=C^{-1} \\
g_{2}=A \\
g_{3}=B A C B\end{array}$ & $\begin{array}{l}\text { (1) } g_{1} g_{2} g_{1}^{-1} g_{2}^{-1}=1 \\
\text { (2) } g_{1} g_{2}^{-1} g_{3}^{-1} g_{1} g_{3} g_{1}^{-1} g_{3} g_{2} g_{1}^{-1} g_{3} g_{1}^{-1} g_{3}^{-1} g_{1} g_{3}^{-1}=1\end{array}$ \\
\hline & \multicolumn{2}{|c|}{ Iso $(H)$-conjugate to $\Gamma_{-7}(6,1), N=3$} \\
\hline \multirow[t]{2}{*}{$\Gamma_{-7}(6,4)$} & $\begin{array}{l}g_{1}=C A^{-1} \\
g_{2}=A^{-2} \\
g_{3}=B C A^{-1} B\end{array}$ & $\begin{array}{l}\text { (1) } g_{1} g_{2} g_{1}^{-1} g_{2}^{-1}=1 \\
\text { (2) } g_{1} g_{2}^{-1} g_{3}^{-1} g_{1} g_{3} g_{1}^{-1} g_{3} g_{2} g_{1}^{-1} g_{3} g_{1}^{-1} g_{3}^{-1} g_{1} g_{3}^{-1}=1\end{array}$ \\
\hline & \multicolumn{2}{|c|}{ Iso $(H)$-conjugate to $\Gamma_{-7}(6,1), N=6$} \\
\hline \multirow[t]{2}{*}{$\Gamma_{-7}(6,5)$} & $\begin{array}{l}g_{1}=C \\
g_{2}=B A B\end{array}$ & $\begin{array}{l}\text { (1) } g_{1} g_{2}^{-1} g_{1}^{-1} g_{2}^{-1} g_{1} g_{2} g_{1}^{-1} g_{2}^{-1} g_{1} g_{2} g_{1} g_{2}^{-1} g_{1}^{-1} g_{2} g_{1}- \\
g_{2} g_{1}^{-1} g_{2}^{-1} g_{1} g_{2} g_{1}^{-1} g_{2}^{-1} g_{1}^{-1} g_{2}=1\end{array}$ \\
\hline & \multicolumn{2}{|c|}{$\Gamma_{-7}(6,5)^{a b} \cong \mathbb{Z}^{2}$, torsion-free, link complement group $(2.2), N=6$} \\
\hline \multirow[t]{2}{*}{$\Gamma_{-7}(6,6)$} & $\begin{array}{l}g_{1}=A C^{-1} \\
g_{2}=B A B\end{array}$ & $\begin{array}{l}\text { (1) } g_{1} g_{2}^{-1} g_{1}^{-1} g_{2}^{-1} g_{1} g_{2} g_{1}^{-1} g_{2}^{-1} g_{1} g_{2} g_{1} g_{2}^{-1} g_{1}^{-1} g_{2} g_{1} g_{2}- \\
g_{1}^{-1} g_{2}^{-1} g_{1} g_{2} g_{1}^{-1} g_{2}^{-1} g_{1}^{-1} g_{2}=1\end{array}$ \\
\hline & \multicolumn{2}{|c|}{ Iso $(H)$-conjugate to $\Gamma_{-7}(6,5), N=3$} \\
\hline \multirow[t]{2}{*}{$\Gamma_{-7}(6,7)$} & $\begin{array}{l}g_{1}=A C^{-1} \\
g_{2}=C^{2} \\
g_{3}=C B C^{-1} B\end{array}$ & $\begin{array}{l}\text { (1) } g_{1} g_{2} g_{1}^{-1} g_{2}^{-1}=1 \\
\text { (2) } g_{1}^{2} g_{2} g_{3}^{-1} g_{1} g_{3}^{-1} g_{1}^{2} g_{2} g_{3}^{-2} g_{1} g_{2} g_{3}^{-2}=1\end{array}$ \\
\hline & \multicolumn{2}{|c|}{$\Gamma_{-7}(6,7)^{a b}=\mathbb{Z}^{2} \times \mathbb{Z} / 3 \mathbb{Z}, N=3$} \\
\hline \multirow[t]{2}{*}{$\Gamma_{-7}(6,8)$} & $\begin{array}{l}g_{1}=A C B \\
g_{2}=A^{2} \\
g_{3}=A B C\end{array}$ & $\begin{array}{l}\text { (1) } g_{1} g_{2}^{-1} g_{3} g_{2}^{-1} g_{3}^{-1} g_{1}^{-1} g_{2}^{-1}=1 \\
\text { (2) } g_{1} g_{2} g_{3}^{-1} g_{1} g_{3}^{-1} g_{2}^{-1} g_{1} g_{3}^{-1}=1\end{array}$ \\
\hline & \multicolumn{2}{|c|}{$\Gamma_{-7}(6,8)^{a b}=\mathbb{Z} \times \mathbb{Z} / 3 \mathbb{Z} \times \mathbb{Z} / 3 \mathbb{Z}, N=2$} \\
\hline \multirow[t]{2}{*}{$\Gamma_{-7}(6,9)$} & $\begin{array}{l}g_{1}=B C^{-1} \\
g_{2}=A^{-1} C^{3} \\
g_{3}=A B C A^{-1}\end{array}$ & $\begin{array}{l}\text { (1) } g_{1}^{2} g_{2} g_{1} g_{3}^{2} g_{2}^{-1} g_{3}=1 \\
\text { (2) } g_{1}^{2} g_{2} g_{3}^{-1} g_{2} g_{3} g_{2}^{-1} g_{1}^{-2} g_{3}^{-1} g_{1}^{-1} g_{2}^{-1} g_{1} g_{3}=1 \\
\text { (3) } g_{1}^{2} g_{2} g_{3}^{-2} g_{1}^{2} g_{2} g_{3}^{-2} g_{1} g_{2} g_{1} g_{3}=1\end{array}$ \\
\hline & \multicolumn{2}{|c|}{$\Gamma_{-7}(6,9)^{a b}=\mathbb{Z} \times \mathbb{Z} / 3 \mathbb{Z} \times \mathbb{Z} / 3 \mathbb{Z}, N=2$} \\
\hline
\end{tabular}


TABLE 2 (continued)

\begin{tabular}{|c|c|c|}
\hline$\Gamma_{-7}(6,10)$ & $\begin{array}{l}g_{1}=C \\
g_{2}=B C^{2} B \\
g_{3}=B A C B A^{-1}\end{array}$ & $\begin{array}{l}\text { (1) } g_{1} g_{2}^{-1} g_{3} g_{1} g_{3}^{-1} g_{2} g_{3} g_{1}^{-1} g_{3}^{-1} g_{2} g_{1}^{-1} g_{2}^{-1}=1 \\
\text { (2) } g_{1}^{2} g_{2}^{-1} g_{1} g_{2}^{-1} g_{3} g_{1}^{2} g_{2}^{-1} g_{3} g_{1} g_{3}^{-1} g_{1} g_{2}^{-1} g_{3} g_{1}^{2} g_{2}^{-1} g_{1}- \\
g_{2}^{-1} g_{3}=1 \\
\text { (3) } g_{1}^{2} g_{2}^{-1} g_{3} g_{1} g_{3}^{-1} g_{1} g_{3} g_{1}^{-1} g_{3}^{-1} g_{2} g_{1}^{-2} g_{3}^{-1} g_{2} g_{1}^{-1} g_{3} g_{1}^{-1}- \\
g_{3}^{-1} g_{1} g_{2}^{-1} g_{3}=1\end{array}$ \\
\hline & \multicolumn{2}{|c|}{$\Gamma_{-7}(6,10)^{a b}=\mathbb{Z}^{2} \times \mathbb{Z} / 3 \mathbb{Z}, N=3$} \\
\hline \multirow[t]{2}{*}{$\Gamma_{-7}(6,11)$} & $\begin{array}{l}g_{1}=A \\
g_{2}=C B C^{-1} B \\
g_{3}=B A^{-1} C^{2} B\end{array}$ & $\begin{array}{l}\text { (1) }\left(g_{1} g_{2}^{-1}\right)^{2}=1 \\
\text { (2) } g_{1} g_{2} g_{3} g_{2}^{-1} g_{1}^{-1} g_{2}^{2} g_{1}^{-1} g_{3}^{-1} g_{1} g_{2}^{-2}=1 \\
\text { (3) } g_{1} g_{2} g_{3} g_{1}^{-1} g_{3}^{-1} g_{1} g_{2}^{-1} g_{3} g_{1}^{-1} g_{3}^{-1} g_{2} g_{1}^{-1} g_{3} g_{1} g_{3}^{-1} g_{2}^{-1}=1\end{array}$ \\
\hline & \multicolumn{2}{|c|}{$\Gamma_{-7}(6,11)^{a b} \cong \mathbb{Z}^{2} \times \mathbb{Z} / 2 \mathbb{Z}$, not torsion-free, $N=1$} \\
\hline \multirow[t]{2}{*}{$\Gamma_{-7}(6,12)$} & $\begin{array}{l}g_{1}=B \\
g_{2}=C^{2} \\
g_{3}=C B A C^{2} B C^{-1} \\
g_{4}=C B A^{2} B C^{-1} \\
g_{5}=A\end{array}$ & $\begin{array}{l}\text { (1) } g_{1}^{2}=1 \\
\text { (2) } g_{2} g_{5} g_{2}^{-1} g_{5}^{-1}=1 \\
\text { (3) }\left(g_{5} g_{4}\right)^{2}=1 \\
\text { (4) } g_{3} g_{4} g_{3}^{-1} g_{4}^{-1}=1 \\
\text { (5) }\left(g_{1} g_{5}\right)^{3}=1 \\
\text { (6) }\left(g_{5} g_{1} g_{4}\right)^{3}=1 \\
\text { (7) } g_{5} g_{1} g_{4} g_{3}^{-1} g_{2} g_{5}^{-1} g_{1}^{-1} g_{2}^{-1} g_{3} g_{4}^{-1}=1\end{array}$ \\
\hline & \multicolumn{2}{|c|}{$\Gamma_{-7}(6,12)^{a b} \cong \mathbb{Z}^{2} \times \mathbb{Z} / 2 \times \mathbb{Z} / 3 \mathbb{Z}$, not torsion-free, $N=1$} \\
\hline \multirow[t]{2}{*}{$\Gamma_{-7}(6,13)$} & $\begin{array}{l}g_{1}=A \\
g_{2}=B \\
g_{3}=C^{3} \\
g_{4}=C^{-1} B A C B C\end{array}$ & $\begin{array}{l}\text { (1) } g_{2}^{2}=1 \\
\text { (2) } g_{1} g_{3} g_{1}^{-1} g_{3}^{-1}=1 \\
\text { (3) }\left(g_{1} g_{2}\right)^{3}=1 \\
\text { (4) } g_{1} g_{4} g_{1} g_{4} g_{1}^{-1} g_{4}^{-1} g_{1}^{-1} g_{2}^{-1} g_{4}^{-1} g_{2}=1 \\
\text { (5) }\left(g_{1}^{2} g_{3}^{-1} g_{2} g_{3} g_{1}^{-1} g_{4}^{-1} g_{1}^{-1} g_{2} g_{1} g_{4}\right)^{2}=1\end{array}$ \\
\hline & \multicolumn{2}{|c|}{$\Gamma_{-7}(6,13)^{a b} \cong \mathbb{Z}^{2} \times \mathbb{Z} / 2 \mathbb{Z}$, not torsion-free, $N=1$} \\
\hline \multirow[t]{2}{*}{$\Gamma_{-7}(6,14)$} & $\begin{array}{l}g_{1}=C \\
g_{2}=A^{3} \\
g_{3}=B\end{array}$ & $\begin{array}{l}\text { (1) } g_{3}^{2}=1 \\
\text { (2) } g_{1} g_{2} g_{1}^{-1} g_{2}^{-1}=1 \\
\text { (3) } g_{1}^{2} g_{3}^{-1} g_{1}^{-1} g_{3}^{-1} g_{1} g_{3} g_{1}^{-1} g_{2} g_{3} g_{1} g_{3} g_{1}^{-1} g_{3}^{-1} g_{1} g_{2}^{-1}- \\
g_{3}^{-1} g_{1}^{-1} g_{3} g_{1} g_{3} g_{1}^{-1} g_{2} g_{1}^{-1} g_{3} g_{1} g_{3} g_{1}^{-1} g_{3}^{-1} g_{2}^{-1} g_{1}- \\
g_{3}^{-1} g_{1}^{-1} g_{3}^{-1} g_{1} g_{3} g_{2} g_{1}^{-1} g_{3} g_{1} g_{3}^{-1} g_{1}^{-1} g_{3}^{-1} g_{2}^{-1}=1\end{array}$ \\
\hline & \multicolumn{2}{|c|}{$\Gamma_{-7}(6,14)^{a b} \cong \mathbb{Z}^{2} \times \mathbb{Z} / 2 \mathbb{Z}$, not torsion-free, $N=1$} \\
\hline \multirow[t]{2}{*}{$\Gamma_{-7}(6,15)$} & $\begin{array}{l}g_{1}=C \\
g_{2}=B \\
g_{3}=A^{-2} B A^{2} \\
g_{4}=A^{4}\end{array}$ & $\begin{array}{l}\text { (1) } g_{2}^{2}=1 \\
\text { (2) } g_{3}^{2}=1 \\
\text { (3) } g_{1} g_{4} g_{1}^{-1} g_{4}^{-1}=1 \\
\text { (4) } g_{1} g_{2} g_{1}^{-1} g_{2}^{-1} g_{1} g_{3}^{-1} g_{1}^{-1} g_{3}^{-1} g_{1} g_{3} g_{1}^{-1} g_{2}=1 \\
\text { (5) } g_{1} g_{2} g_{1}^{-1} g_{4} g_{3} g_{1} g_{3}^{-1} g_{1}^{-1} g_{3}^{-1} g_{1} g_{4}^{-1} g_{2}^{-1} g_{1}^{-1} g_{2}=1\end{array}$ \\
\hline & \multicolumn{2}{|c|}{$\Gamma_{-7}(6,15)^{a b} \cong \mathbb{Z}^{2} \times \mathbb{Z} / 2 \mathbb{Z}$, not torsion-free, $N=3$} \\
\hline
\end{tabular}


TABLE 2 (continued)

\begin{tabular}{|c|c|c|}
\hline$\Gamma_{-7}(6,16)$ & $\begin{array}{l}g_{1}=A \\
g_{2}=C B C^{-1} B \\
g_{3}=B C^{2} B\end{array}$ & $\begin{array}{l}\text { (1) }\left(g_{1} g_{2}^{-1}\right)^{2}=1 \\
\text { (2) } g_{1} g_{2} g_{3} g_{2}^{-1} g_{1}^{-1} g_{2}^{2} g_{1}^{-1} g_{3}^{-1} g_{1} g_{2}^{-2}=1 \\
\text { (3) } g_{1} g_{2} g_{3} g_{1} g_{3}^{-1} g_{2}^{-1} g_{1}^{-1} g_{2} g_{1} g_{2}^{-2} g_{1} g_{2} g_{3} g_{1}^{-1} g_{3}^{-1} g_{2}^{-1}- \\
g_{1}^{-1} g_{2} g_{3} g_{1} g_{3}^{-1} g_{2}^{-1} g_{1}^{-1} g_{2}^{2} g_{1}^{-1} g_{2}^{-1}=1\end{array}$ \\
\hline & \multicolumn{2}{|c|}{$\Gamma_{-7}(6,16)^{a b} \cong \mathbb{Z}^{2} \times \mathbb{Z} / 2 \mathbb{Z}$, not torsion-free, $N=3$} \\
\hline \multirow[t]{2}{*}{$\Gamma_{-7}(6,17)$} & $\begin{array}{l}g_{1}=C \\
g_{2}=A^{2} \\
g_{3}=A B C^{-1} B \\
g_{4}=B C^{2} B\end{array}$ & $\begin{array}{l}\text { (1) } g_{1} g_{2} g_{1}^{-1} g_{2}^{-1}=1 \\
\text { (2) }\left(g_{1} g_{2}^{-1} g_{3}\right)^{2}=1 \\
\text { (3) }\left(g_{1} g_{3} g_{2}^{-1}\right)^{2}=1 \\
\text { (4) }\left(g_{1} g_{4}^{-1} g_{3}^{-1}\right)^{2}=1 \\
\text { (5) } g_{1} g_{3} g_{2}^{-1} g_{3}^{2} g_{4} g_{1}^{-1} g_{3}^{-1} g_{2} g_{3}^{-1} g_{4}^{-1} g_{3}^{-1}=1 \\
\text { (6) } g_{1} g_{4}^{-1} g_{3}^{-1} g_{4} g_{3} g_{4} g_{1}^{-1} g_{3}^{-1} g_{2} g_{4}^{-1} g_{2}^{-1} g_{3}=1\end{array}$ \\
\hline & \multicolumn{2}{|c|}{$\Gamma_{-7}(6,17)^{a b} \cong \mathbb{Z}^{2} \times \mathbb{Z} / 2 \mathbb{Z} \times \mathbb{Z} / 2 \mathbb{Z}$, not torsion-free, $N=3$} \\
\hline \multirow[t]{2}{*}{$\Gamma_{-7}(6,18)$} & $\begin{array}{l}g_{1}=A \\
g_{2}=B C^{2} B \\
g_{3}=B C B C^{-1} B\end{array}$ & $\begin{array}{l}\text { (1) } g_{3}^{2}=1 \\
\text { (2) } g_{1} g_{3} g_{1}^{-1} g_{3} g_{2} g_{3}^{-1} g_{1} g_{3}^{-1} g_{1}^{-1} g_{3} g_{1}^{-1} g_{2}^{-1} g_{1} g_{3}^{-1}=1 \\
\text { (3) } g_{1} g_{2}^{-1} g_{3}^{-1} g_{1} g_{3}^{-1} g_{1} g_{3} g_{1}^{-1} g_{3} g_{2} g_{1}^{-1} g_{2}^{-1} g_{3} g_{2} g_{1}^{-1}- \\
g_{2}^{-1} g_{3}^{-1} g_{2}=1\end{array}$ \\
\hline & \multicolumn{2}{|c|}{$\Gamma_{-7}(6,18)^{a b} \cong \mathbb{Z}^{2} \times \mathbb{Z} / 2 \mathbb{Z}$, not torsionfree, $N=3$} \\
\hline \multirow[t]{2}{*}{$\Gamma_{-7}(6,19)$} & $\begin{array}{l}g_{1}=B A C^{-1} B \\
g_{2}=B C^{2} B A^{-1} \\
g_{3}=A B C^{2} B \\
g_{4}=A^{2}\end{array}$ & $\begin{array}{l}\text { (1) }\left(g_{1}^{2} g_{3}\right)^{2}=1 \\
\text { (2) } g_{1} g_{2} g_{3} g_{1}^{-1} g_{3}^{-1} g_{2}^{-1}=1 \\
\text { (3) }\left(g_{4} g_{1}^{2} g_{2}\right)^{2}=1 \\
\text { (4) } g_{4} g_{1} g_{3} g_{1} g_{3} g_{1} g_{3}^{-1} g_{4}^{-1} g_{3} g_{1}^{-1} g_{3}^{-1} g_{1}^{-1} g_{3}^{-1} g_{1}^{-1}=1 \\
\text { (5) } g_{4} g_{1} g_{2} g_{4} g_{1} g_{3} g_{1}^{-1} g_{3}^{-1} g_{1}^{-1} g_{3}^{-1} g_{1}^{-1} g_{4}^{-1} g_{2}^{-1} g_{1} g_{2}=1\end{array}$ \\
\hline & \multicolumn{2}{|c|}{$\Gamma_{-7}(6,19)^{a b} \cong \mathbb{Z}^{2} \times \mathbb{Z} / 2 \mathbb{Z}$, not torsion-free, $N=3$} \\
\hline \multirow[t]{2}{*}{$\Gamma_{-7}(6,20)$} & $\begin{array}{l}g_{1}=A C^{-1} \\
g_{2}=C^{2} \\
g_{3}=B A C B \\
g_{4}=B A C^{-1} B C^{-1}\end{array}$ & $\begin{array}{l}\text { (1) } g_{1} g_{2} g_{1}^{-1} g_{2}^{-1}=1 \\
\text { (2) }\left(g_{2} g_{4}\right)^{2}=1 \\
\text { (3) }\left(g_{1} g_{2} g_{3} g_{4}\right)^{2}=1 \\
\text { (4) } g_{1}^{2} g_{4}^{-1} g_{2}^{-1} g_{1}^{-1} g_{4}^{-1} g_{3}^{-1} g_{4} g_{1}^{-2} g_{4} g_{1} g_{2} g_{3}=1 \\
\text { (5) } g_{1} g_{2} g_{3} g_{1} g_{4}^{-1} g_{3} g_{4} g_{1}^{-1} g_{3}^{-1} g_{2}^{-1} g_{1}^{-1} g_{4}^{-1} g_{1} g_{3}^{-1} g_{1}^{-1} g_{4}=1\end{array}$ \\
\hline & \multicolumn{2}{|c|}{$\Gamma_{-7}(6,20)^{a b} \cong \mathbb{Z}^{2} \times \mathbb{Z} / 2 \mathbb{Z} \times \mathbb{Z} / 2 \mathbb{Z}$, not torsion-free, $N=3$} \\
\hline$\Gamma_{-7}(6,21)$ & $\begin{array}{l}g_{1}=B \\
g_{2}=A^{-1} C^{2} \\
g_{3}=A^{2} C^{2} \\
g_{4}=A B A C\end{array}$ & $\begin{array}{l}\text { (1) } g_{1}^{2}=1 \\
\text { (2) } g_{2} g_{3} g_{2}^{-1} g_{3}^{-1}=1 \\
\text { (3) }\left(g_{1} g_{4} g_{1} g_{4} g_{2}^{-1}\right)^{2}=1 \\
\text { (4) }\left(g_{2} g_{3}^{-1} g_{4} g_{3}^{-1} g_{4}\right)^{2}=1 \\
\text { (5) } g_{1} g_{2} g_{1}^{-1} g_{4}^{-1} g_{1}^{-1} g_{2} g_{4}^{-1} g_{2}^{-1} g_{4} g_{1} g_{4} g_{2}^{-1}=1 \\
\text { (6) } g_{1} g_{2} g_{4}^{-1} g_{1}^{-1} g_{2} g_{3}^{-1} g_{4} g_{3}^{-1} g_{4} g_{1} g_{4} g_{2}^{-1}=1\end{array}$ \\
\hline & \multicolumn{2}{|c|}{$\Gamma_{-7}(6,21)^{a b} \cong \mathbb{Z} \times \mathbb{Z} / 2 \mathbb{Z} \times \mathbb{Z} / 4 \mathbb{Z}$, not torsion-free, $N=3$} \\
\hline
\end{tabular}


TABLE 2 (continued)

\begin{tabular}{|c|c|c|}
\hline$\Gamma_{-7}(6,22)$ & $\begin{array}{l}g_{1}=B A^{-1} \\
g_{2}=C B A^{-1} C^{-1} \\
g_{3}=C^{-1} B A^{-1} C \\
g_{4}=C^{3}\end{array}$ & $\begin{array}{l}\text { (1) } g_{1}^{3}=1 \\
\text { (2) } g_{2}^{3}=1 \\
\text { (3) } g_{3}^{3}=1 \\
\text { (4) } g_{1} g_{2} g_{1}^{-1} g_{3} g_{1}^{-1} g_{3}^{-1} g_{1} g_{2}^{-1}=1 \\
\text { (5) } g_{1} g_{4} g_{1}^{-1} g_{3} g_{1} g_{3}^{-1} g_{4}^{-1} g_{3} g_{1}^{-1} g_{3}^{-1}=1 \\
\text { (6) } g_{1} g_{3}^{-1} g_{4}^{-1} g_{2} g_{4} g_{3} g_{4}^{-1} g_{2}^{-1} g_{4} g_{3} g_{1}^{-1} g_{3}^{-1}=1\end{array}$ \\
\hline & \multicolumn{2}{|c|}{$\Gamma_{-7}(6,22)^{a b} \cong \mathbb{Z} \times \mathbb{Z} / 3 \mathbb{Z} \times \mathbb{Z} / 3 \mathbb{Z} \times \mathbb{Z} / 3 \mathbb{Z}$, not torsion-free, $N=3$} \\
\hline \multirow[t]{2}{*}{$\Gamma_{-7}(6,23)$} & $\begin{array}{l}g_{1}=B A^{-1} \\
g_{2}=C B A^{-1} C^{-1} \\
g_{3}=C^{3} A^{-1} \\
g_{4}=A C^{-1} B C\end{array}$ & $\begin{array}{l}\text { (1) } g_{1}^{3}=1 \\
\text { (2) } g_{2}^{3}=1 \\
\text { (3) } g_{4}^{3}=1 \\
\text { (4) } g_{1} g_{2} g_{1}^{-1} g_{4}^{-1} g_{1}^{-1} g_{4} g_{1} g_{2}^{-1}=1 \\
\text { (5) } g_{1} g_{3} g_{1}^{-1} g_{4}^{-1} g_{1} g_{4} g_{3}^{-1} g_{4}^{-1} g_{1}^{-1} g_{4}=1 \\
\text { (6) } g_{1} g_{2} g_{1}^{-1} g_{3} g_{4}^{-1} g_{3}^{-1} g_{2}^{-1} g_{3} g_{4} g_{3}^{-1}=1\end{array}$ \\
\hline & \multicolumn{2}{|c|}{$\Gamma_{-7}(6,23)^{a b} \cong \mathbb{Z} \times \mathbb{Z} / 3 \mathbb{Z} \times \mathbb{Z} / 3 \mathbb{Z} \times \mathbb{Z} / 3 \mathbb{Z}$, not torsion-free, $N=3$} \\
\hline \multirow[t]{2}{*}{$\Gamma_{-7}(6,24)$} & $\begin{array}{l}g_{1}=A \\
g_{2}=C^{2} \\
g_{3}=B \\
g_{4}=C^{-1} B C^{2} B C\end{array}$ & $\begin{array}{l}\text { (1) } g_{3}^{2}=1 \\
\text { (2) }\left(g_{1} g_{3}\right)^{3}=1 \\
\text { (3) } g_{1} g_{2} g_{1}^{-1} g_{2}^{-1}=1 \\
\text { (4) }\left(g_{4} g_{2}^{-1} g_{3} g_{1}^{-1} g_{2} g_{4}^{-1} g_{1}^{-1} g_{3} g_{1}\right)^{2}=1 \\
\text { (5) } g_{2}^{-1} g_{3} g_{1}^{-1} g_{2} g_{4}^{-1} g_{1}^{-1} g_{3} g_{4}^{-1} g_{3} g_{1} g_{4} g_{2}^{-1} g_{1} g_{3} g_{2} g_{4}=1\end{array}$ \\
\hline & \multicolumn{2}{|c|}{$\Gamma_{-7}(6,24)^{a b} \cong \mathbb{Z}^{2} \times \mathbb{Z} / 2 \mathbb{Z}$, not torsionfree, $N=1$} \\
\hline$\Gamma_{-7}(6,25)$ & $\begin{array}{l}g_{1}=A \\
g_{2}=C^{6} \\
g_{3}=B \\
g_{4}=C B C^{-1} \\
g_{5}=C^{-1} B C \\
g_{6}=C^{2} B C^{-2} \\
g_{7}=C^{-2} B C^{2} \\
g_{8}=C^{3} B C^{-3}\end{array}$ & $\begin{array}{l}\text { (1) } \begin{array}{l}g_{3}^{2}=g_{4}^{2}= \\
\text { (2) } \begin{aligned}\left(g_{1} g_{3}\right)^{3}= & =\left(g_{1} g_{4}\right)^{3}=\left(g_{1} g_{5}\right)^{3}=\left(g_{1} g_{6}\right)^{3} \\
& =\left(g_{1} g_{7}\right)^{3}=\left(g_{1} g_{8}\right)^{3}=1\end{aligned} \\
\text { (3) } \begin{aligned}\left(g_{1} g_{5} g_{3}\right)^{2} & =\left(g_{1} g_{3} g_{4}\right)^{2}=\left(g_{1} g_{4} g_{6}\right)^{2}=\left(g_{1} g_{6} g_{8}\right)^{2} \\
& =\left(g_{1} g_{8} g_{2} g_{7} g_{2}^{-1}\right)^{2}=\left(g_{1} g_{7} g_{5}\right)^{2}=1\end{aligned} \\
\text { (4) } g_{1} g_{2} g_{1}^{-1} g_{2}^{-1}=1\end{array}\end{array}$ \\
\hline & \multicolumn{2}{|c|}{$\Gamma_{-7}(6,25)^{a b} \cong \mathbb{Z} \times \mathbb{Z} / 2 \mathbb{Z}$, not torsion-free, $N=6$} \\
\hline
\end{tabular}




\section{TABLE 3}

All subgroups of index 12 in $\Gamma_{-7}$ with torsion-free commutator factorgroup, up to $\Gamma_{-7}$ conjugacy

\begin{tabular}{|c|c|c|}
\hline & Generators & Relations \\
\hline \multirow[t]{2}{*}{$\Gamma_{-7}(12,1)$} & $\begin{array}{l}g_{1}=C \\
g_{2}=A \\
g_{3}=B C^{2} B \\
g_{4}=B C A^{3} B\end{array}$ & $\begin{array}{l}\text { (1) } g_{1} g_{2} g_{1}^{-1} g_{2}^{-1}=1 \\
\text { (2) } g_{3} g_{4} g_{3}^{-1} g_{4}^{-1}=1 \\
\text { (3) } g_{1} g_{3}^{-1} g_{2}^{-1} g_{3} g_{4}^{-1} g_{2}^{-1} g_{4}^{-1} g_{2}^{-1} g_{3} g_{4}^{-1} g_{1}^{-1} g_{3} g_{2} g_{4} g_{3}^{-1}- \\
g_{2} g_{4} g_{2} g_{4} g_{3}^{-1}=1\end{array}$ \\
\hline & \multicolumn{2}{|c|}{$\Gamma_{-7}(12,1)^{a b}=\mathbb{Z}^{4}$, generated by unipotent elements, $N=12$} \\
\hline \multirow[t]{2}{*}{$\Gamma_{-7}(12,2)$} & $\begin{array}{l}g_{1}=C \\
g_{2}=A \\
g_{3}=B C^{3} B \\
g_{4}=B C A^{2} B\end{array}$ & $\begin{array}{l}\text { (1) } g_{1} g_{2} g_{1}^{-1} g_{2}^{-1}=1 \\
\text { (2) } g_{3} g_{4} g_{3}^{-1} g_{4}^{-1}=1 \\
\text { (3) } g_{1} g_{2}^{-1} g_{4} g_{3}^{-1} g_{2} g_{4} g_{2} g_{4}^{2} g_{3}^{-1} g_{2} g_{4} g_{2} g_{1}^{-1} g_{3} g_{4}^{-1} g_{2}^{-1}- \\
g_{4}^{-1} g_{2}^{-1} g_{3} g_{4}^{-2} g_{2}^{-1} g_{4}^{-1}=1\end{array}$ \\
\hline & \multicolumn{2}{|c|}{$\Gamma_{-7}(12,2)^{a b}=\mathbb{Z}^{4}$, generated by unipotent elements, $N=12$} \\
\hline \multirow[t]{2}{*}{$\Gamma_{-7}(12,3)$} & $\begin{array}{l}g_{1}=C \\
g_{2}=A \\
g_{3}=B C^{4} B \\
g_{4}=B C B C^{-1} B C^{-1} B\end{array}$ & $\begin{array}{l}\text { (1) } g_{1} g_{2} g_{1}^{-1} g_{2}^{-1}=1 \\
\text { (2) } g_{1} g_{2}^{-1} g_{3} g_{2} g_{1}^{-1} g_{4}^{-1} g_{2} g_{4}^{-1} g_{3}^{-1} g_{4} g_{2}^{-1} g_{4}=1 \\
\text { (3) } g_{1} g_{2}^{-2} g_{3}^{-1} g_{4} g_{2}^{-1} g_{4} g_{1} g_{2}^{-1} g_{4}^{-1} g_{3} g_{2} g_{1}^{-1} g_{4}^{-1} g_{2} g_{4}^{-1}- \\
g_{2}^{2} g_{1}^{-1} g_{4}^{-1} g_{2} g_{4}^{-1} g_{3} g_{2} g_{1}^{-1} g_{4} g_{1} g_{2}^{-1} g_{3} g_{4} g_{2}^{-1} g_{4}=1\end{array}$ \\
\hline & \multicolumn{2}{|c|}{$\Gamma_{-7}(12,3)^{a b}=\mathbb{Z}^{4}$, contained in $\Gamma_{-7}(6,2)$, Iso $(H)$-conjugate to $\Gamma_{-7}(12,4)$} \\
\hline \multirow[t]{2}{*}{$\Gamma_{-7}(12,4)$} & $\begin{array}{l}g_{1}=C \\
g_{2}=B C^{2} B \\
g_{3}=B C A^{2} B \\
g_{4}=A B C^{2} B A^{-1}\end{array}$ & $\begin{array}{l}\text { (1) } g_{2} g_{3}^{-1} g_{2}^{-1} g_{3}^{-1}=1 \\
\text { (2) } g_{1} g_{2}^{-1} g_{3} g_{4} g_{1}^{-1} g_{4} g_{1} g_{4}^{-1} g_{3}^{-1} g_{2} g_{1}^{-1} g_{2} g_{4}^{-1} g_{2}^{-1}=1 \\
\text { (3) } g_{1} g_{2}^{-2} g_{3} g_{1}^{-1} g_{2}^{-1} g_{3} g_{4} g_{1}^{-1} g_{3}^{-1} g_{2} g_{1}^{-1} g_{2} g_{4}^{-1} g_{3}^{-1} g_{2}- \\
g_{1}^{-1} g_{3}^{-1} g_{2} g_{1} g_{2}^{-1} g_{3}=1\end{array}$ \\
\hline & \multicolumn{2}{|c|}{$\begin{array}{l}\Gamma_{-7}(12,4)^{a b} \cong \mathbb{Z}^{4}, \text { link complement group }(2.3) \text {, contained in } \Gamma_{-7}(6,1) \text { and } \\
\Gamma_{-7}(6,2)\end{array}$} \\
\hline \multirow[t]{2}{*}{$\Gamma_{-7}(12,5)$} & $\begin{array}{l}g_{1}=A C^{-1} \\
g_{2}=C^{2} \\
g_{3}=B A C B \\
g_{4}=A^{-1} B A C B A\end{array}$ & $\begin{array}{l}\text { (1) } g_{1} g_{2} g_{1}^{-1} g_{2}^{-1}=1 \\
\text { (2) } g_{4} g_{2}^{-1} g_{1}^{-1} g_{4} g_{3} g_{4}^{-1} g_{1} g_{2} g_{4}^{-1} g_{3}^{-1}=1 \\
\text { (3) } g_{1}^{2} g_{2} g_{4}^{-1} g_{3}^{-1} g_{1}^{-1} g_{3}^{-1} g_{1}^{-1} g_{2}^{-1} g_{1}^{-1} g_{4} g_{3} g_{1} g_{3}=1\end{array}$ \\
\hline & \multicolumn{2}{|c|}{$\begin{array}{l}\Gamma_{-7}(12,5)^{a b} \cong \mathbb{Z}^{4}, \text { Iso }(H) \text {-conjugate to } \Gamma_{-7}(12,4), \text { contained in } \Gamma_{-7}(6,3) \text { and } \\
\Gamma_{-7}(6,4)\end{array}$} \\
\hline \multirow[t]{2}{*}{$\Gamma_{-7}(12,6)$} & $\begin{array}{l}g_{1}=C \\
g_{2}=A \\
g_{3}=B C^{2} A^{2} B \\
g_{4}=B C B C B\end{array}$ & $\begin{array}{l}\text { (1) } g_{1} g_{2} g_{1}^{-1} g_{2}^{-1}=1 \\
\text { (2) } g_{1} g_{2}^{-1} g_{4}^{-1} g_{3} g_{4} g_{2} g_{1}^{-1} g_{4} g_{3}^{-1} g_{4}^{-1}=1 \\
\text { (3) } g_{1} g_{2}^{-1} g_{4}^{-1} g_{3} g_{2} g_{3} g_{4}^{-1} g_{1}^{-1} g_{3}^{-1} g_{4} g_{2} g_{1}^{-1} g_{4} g_{2}^{-1} g_{3}^{-1}- \\
g_{4} g_{2} g_{4}^{-1}=1\end{array}$ \\
\hline & \multicolumn{2}{|c|}{$\Gamma_{-7}(12,6)^{a b} \cong \mathbb{Z}^{4}$, Iso $(H)$-conjugate to $\Gamma_{-7}(12,4)$, contained in $\Gamma_{-7}(6,3)$} \\
\hline
\end{tabular}


TABLE 3 (continued)

\begin{tabular}{|c|c|c|}
\hline$\Gamma_{-7}(12,7)$ & $\begin{array}{l}g_{1}=C \\
g_{2}=A^{3} \\
g_{3}=B C B \\
g_{4}=A^{-1} B A^{3} B A\end{array}$ & $\begin{array}{l}\text { (1) } g_{1} g_{2} g_{1}^{-1} g_{2}^{-1}=1 \\
\text { (2) } g_{1} g_{2}^{-1} g_{3}^{-1} g_{1} g_{3}^{-1} g_{4}^{-1} g_{1} g_{2}^{-1} g_{3}^{-1} g_{1} g_{3}^{-1} g_{4}^{-1} g_{2}^{-1} g_{3}^{-1}- \\
g_{1} g_{3}^{-1} g_{4}^{-1} g_{3} g_{1}^{-1} g_{3} g_{1} g_{3}^{-1}=1 \\
\text { (3) } g_{1} g_{2}^{-1} g_{3}^{-1} g_{1} g_{3}^{-1} g_{4}^{-1} g_{2}^{-1} g_{1} g_{3}^{-1} g_{1} g_{4}^{-1} g_{2}^{-1} g_{3}^{-1} g_{1}- \\
g_{3}^{-1} g_{4}^{-1}=1\end{array}$ \\
\hline & \multicolumn{2}{|c|}{$\Gamma_{-7}(12,7)^{a b} \cong \mathbb{Z}^{3}$, generated by unipotent elements, $N=6$} \\
\hline \multirow[t]{2}{*}{$\Gamma_{-7}(12,8)$} & $\begin{array}{l}g_{1}=C \\
g_{2}=B A C^{-2} B \\
g_{3}=B A C B\end{array}$ & $\begin{array}{l}\text { (1) } g_{1} g_{3} g_{1} g_{3} g_{1} g_{3}^{-2} g_{1}^{-1} g_{2}^{-1} g_{3}^{2} g_{1}^{-1} g_{3}^{-1} g_{1}^{-1} g_{3}^{-1} g_{1}^{-1} g_{3}^{2}- \\
g_{1} g_{3}^{-2} g_{2}=1 \\
\text { (2) } g_{1} g_{2} g_{1} g_{2} g_{1}^{-1} g_{2}^{-1} g_{1}^{-1} g_{2}^{-1} g_{3}^{2} g_{1}^{-1} g_{3}^{-1} g_{1}^{-1} g_{3}^{-1} g_{1}^{-1}- \\
g_{2}^{-1} g_{1} g_{3} g_{1} g_{3} g_{1} g_{3}^{-2} g_{2}=1\end{array}$ \\
\hline & \multicolumn{2}{|c|}{$\Gamma_{-7}(12,8)^{a b} \cong \mathbb{Z}^{3}$, generated by unipotent elements, $N=12$} \\
\hline \multirow[t]{2}{*}{$\Gamma_{-7}(12,9)$} & $\begin{array}{l}g_{1}=C \\
g_{2}=B A C B \\
g_{3}=A B C^{-3} A B\end{array}$ & $\begin{array}{l}\text { (1) } g_{1} g_{2} g_{3} g_{2}^{2} g_{3} g_{2} g_{3}^{-1} g_{1}^{-1} g_{3} g_{2}^{-1} g_{3}^{-1} g_{2}^{-2} g_{3}^{-1} g_{2}^{-1}=1 \\
\text { (2) } g_{1} g_{2} g_{1}^{-1} g_{2} g_{3} g_{2}^{2} g_{1} g_{2}^{-1} g_{1}^{-1} g_{2} g_{3} g_{2}^{2} g_{1} g_{3} g_{2}^{-1} g_{3}^{-1}- \\
g_{2}^{-2} g_{3}^{-1} g_{1}^{-1} g_{2}^{-2} g_{3}^{-1} g_{2}^{-1}=1\end{array}$ \\
\hline & \multicolumn{2}{|c|}{$\Gamma_{-7}(12,9)^{a b} \cong \mathbb{Z}^{3}$, generated by unipotent elements, $N=6$} \\
\hline \multirow[t]{2}{*}{$\Gamma_{-7}(12,10)$} & $\begin{array}{l}g_{1}=A C^{-1} \\
g_{2}=B A C^{-1} B \\
g_{3}=B C^{2} B C^{2}\end{array}$ & $\begin{array}{l}\text { (1) } g_{1}^{2} g_{2} g_{1}^{-1} g_{2} g_{1} g_{2}^{-1} g_{1}^{-1} g_{3}^{-1} g_{2}^{-2} g_{1}^{-1} g_{2} g_{1}^{-1} g_{2}^{-1} g_{1} g_{2} g_{3}=1 \\
\text { (2) } g_{1} g_{2} g_{3} g_{1} g_{3}^{-1} g_{2}^{-1} g_{3} g_{1} g_{3}^{-1} g_{2} g_{3} g_{1}^{-1} g_{3}^{-1} g_{2}^{-1} g_{1}^{-1} g_{2}- \\
g_{1}^{-1} g_{2}^{-1}=1\end{array}$ \\
\hline & \multicolumn{2}{|c|}{$\Gamma_{-7}(12,10)^{a b} \cong \mathbb{Z}^{3}$, Iso $(H)$-conjugate to $\Gamma_{-7}(12,12)$, contained in $\Gamma_{-7}(6,4)$} \\
\hline \multirow[t]{2}{*}{$\Gamma_{-7}(12,11)$} & $\begin{array}{l}g_{1}=C \\
g_{2}=B C^{2} A^{-1} B \\
g_{3}=B C B A^{-2} B\end{array}$ & $\begin{array}{l}\text { (1) } g_{1} g_{2}^{-1} g_{1} g_{2}^{-1} g_{3} g_{2} g_{3}^{-1} g_{1}^{-1} g_{3} g_{2}^{-1} g_{3}^{-1} g_{2} g_{1}^{-1} g_{2}=1 \\
\text { (2) } g_{1} g_{2}^{-1} g_{3} g_{2} g_{3}^{-3} g_{2} g_{1}^{-1} g_{3} g_{2} g_{3}^{-1} g_{1} g_{2}^{-1} g_{3}^{2} g_{1}^{-1} g_{3}- \\
g_{2}^{-1} g_{3}^{-1}=1\end{array}$ \\
\hline & \multicolumn{2}{|c|}{$\Gamma_{-7}(12,11)^{a b} \cong \mathbb{Z}^{3}, N=12$} \\
\hline \multirow[t]{2}{*}{$\Gamma_{-7}(12,12)$} & $\begin{array}{l}g_{1}=C \\
g_{2}=B C B \\
g_{3}=A^{-1} B C B A^{-1}\end{array}$ & $\begin{array}{l}\text { (1) } g_{1} g_{2} g_{1}^{-1} g_{2} g_{3}^{-1} g_{1}^{-1} g_{2} g_{1}^{-1} g_{2}^{-1} g_{1} g_{2}^{-1} g_{3} g_{1} g_{2}^{-1}=1 \\
\text { (2) } g_{1} g_{2} g_{1}^{-1} g_{2} g_{3}^{-1} g_{1}^{-1} g_{3} g_{1} g_{3}^{-1} g_{1} g_{3} g_{2}^{-1} g_{1} g_{2}^{-1} g_{1}^{-1} g_{2}- \\
g_{3}^{-1} g_{1}^{-1} g_{3} g_{2}^{-1}=1\end{array}$ \\
\hline & \multicolumn{2}{|c|}{$\begin{array}{l}\Gamma_{-7}(12,12)^{a b} \cong \mathbb{Z}^{3}, \text { link complement group }(2.3), \text { contained in } \Gamma_{-7}(6,1) \\
N=3\end{array}$} \\
\hline \multirow[t]{2}{*}{$\Gamma_{-7}(12,13)$} & $\begin{array}{l}g_{1}=A \\
g_{2}=B C^{2} B \\
g_{3}=C B C A^{2} B\end{array}$ & $\begin{array}{l}\text { (1) } g_{1} g_{2} g_{1}^{-1} g_{2} g_{3}^{-1} g_{2} g_{3}^{-1} g_{1}^{-1} g_{3} g_{2}^{-1} g_{3}^{-1} g_{1} g_{3} g_{2}^{-1} g_{3} g_{2}^{-1}=1 \\
\text { (2) } g_{1} g_{2} g_{1} g_{2}^{-1} g_{1}^{-1} g_{2} g_{3}^{-1} g_{2}^{2} g_{3}^{-1} g_{2} g_{3}^{-1} g_{1}^{-1} g_{3} g_{2}^{-2}- \\
g_{3} g_{2}^{-1} g_{1} g_{2} g_{1}^{-1} g_{2}^{-1} g_{3} g_{2}^{-1}=1 \\
\end{array}$ \\
\hline & \multicolumn{2}{|c|}{$\Gamma_{-7}(12,13)^{a b} \cong \mathbb{Z}^{3}$, contained in $\Gamma_{-7}(6,2)$ and $\Gamma_{-7}(6,5)$} \\
\hline \multirow[t]{2}{*}{$\Gamma_{-7}(12,14)$} & $\begin{array}{l}g_{1}=A \\
g_{2}=C^{2} \\
g_{3}=C B C B C^{2} B \\
g_{4}=B A C B\end{array}$ & $\begin{array}{l}\text { (1) } g_{1} g_{2} g_{1}^{-1} g_{2}^{-1}=1 \\
\text { (2) } g_{1} g_{2}^{-1} g_{3} g_{4} g_{3}^{-1} g_{1}^{-1} g_{2} g_{4}^{-1} g_{1}^{-1} g_{4}^{-1} g_{1} g_{4}=1 \\
\text { (3) } g_{1} g_{4} g_{1} g_{4} g_{3}^{-1} g_{1} g_{3} g_{4}^{-2} g_{1}^{-1} g_{4}^{-1} g_{3} g_{4}^{-2} g_{1}^{-1} g_{4}^{-1}=1\end{array}$ \\
\hline & \multicolumn{2}{|c|}{$\Gamma_{-7}(12,14)^{a b} \cong \mathbb{Z}^{3}, N=12$} \\
\hline
\end{tabular}


TABLE 3 (continued)

\begin{tabular}{|c|c|c|}
\hline$\Gamma_{-7}(12,15)$ & $\begin{array}{l}g_{1}=A \\
g_{2}=C^{2} \\
g_{3}=B C^{-1} A^{2} B \\
g_{4}=C B C^{2} B\end{array}$ & $\begin{array}{l}\text { (1) } g_{1} g_{2} g_{1}^{-1} g_{2}^{-1}=1 \\
\text { (2) } g_{1} g_{4} g_{3} g_{1} g_{3} g_{4} g_{3} g_{4}^{-1}=1 \\
\text { (3) } g_{1} g_{4} g_{3} g_{4}^{-1} g_{2} g_{3} g_{1} g_{3} g_{1}^{-1} g_{3}^{-1} g_{2}^{-1} g_{4} g_{1} g_{3}=1\end{array}$ \\
\hline & \multicolumn{2}{|c|}{$\begin{array}{l}\Gamma_{-7}(12,15)^{a b} \cong \mathbb{Z}^{3}, \text { Iso }(H) \text {-conjugate to } \Gamma_{-7}(12,12), \text { contained in } \Gamma_{-7}(6,2) \\
N=6\end{array}$} \\
\hline \multirow[t]{2}{*}{$\Gamma_{-7}(12,16)$} & $\begin{array}{l}g_{1}=A \\
g_{2}=C B A C B \\
g_{3}=B A C B C^{-1}\end{array}$ & $\begin{array}{l}\text { (1) } g_{1} g_{2} g_{3} g_{1}^{-1} g_{3}^{-1} g_{1}^{-1} g_{3}^{-1} g_{1}^{-1} g_{2}^{-1} g_{3}^{-1} g_{1} g_{3} g_{1} g_{3}=1 \\
\text { (2) } g_{1} g_{2} g_{1} g_{2}^{-1} g_{1}^{-1} g_{3}^{-1} g_{1} g_{3} g_{1} g_{3} g_{1} g_{2} g_{1}^{-1} g_{2}^{-1} g_{1}^{-1} g_{2}- \\
g_{1} g_{2}^{-1} g_{1}^{-1} g_{3}^{-1} g_{1}^{-1} g_{3}^{-1} g_{1}^{-1} g_{3}=1\end{array}$ \\
\hline & \multicolumn{2}{|c|}{$\Gamma_{-7}(12,16)^{a b} \cong \mathbb{Z}^{3}$, Iso $(H)$-conjugate to $\Gamma_{-7}(12,12)$, contained in $\Gamma_{-7}(6,3)$} \\
\hline \multirow[t]{2}{*}{$\Gamma_{-7}(12,17)$} & $\begin{array}{l}g_{1}=A \\
g_{2}=C B A C B \\
g_{3}=B A^{-1} C^{2} B\end{array}$ & $\begin{array}{l}\text { (1) } g_{1} g_{2} g_{1} g_{3}^{-1} g_{1}^{-1} g_{3} g_{2}^{-1} g_{3} g_{1}^{-1} g_{3}^{-1} g_{2} g_{3}^{-1} g_{1} g_{3} g_{1}^{-1}- \\
g_{3}^{-1}=1 \\
\text { (2) } g_{1} g_{2} g_{3}^{-1} g_{2} g_{3}^{-1} g_{1} g_{3} g_{1}^{-1} g_{3} g_{2}^{-1} g_{3} g_{2}^{-1} g_{1}^{-1} g_{2} g_{3}^{-1}- \\
g_{2}^{-1}=1\end{array}$ \\
\hline & \multicolumn{2}{|c|}{$\Gamma_{-7}(12,17)^{a b} \cong \mathbb{Z}^{3}, N=6$} \\
\hline \multirow[t]{2}{*}{$\Gamma_{-7}(12,18)$} & $\begin{array}{l}g_{1}=A C^{-1} \\
g_{2}=C B C^{-1} B \\
g_{3}=B A^{3} B\end{array}$ & $\begin{array}{l}\text { (1) } g_{1} g_{2} g_{3} g_{2}^{-1} g_{1}^{-1} g_{2} g_{1}^{-1} g_{3}^{-1} g_{1} g_{2}^{-1}=1 \\
\text { (2) } g_{1} g_{2}^{2} g_{3} g_{2} g_{1}^{-1} g_{3} g_{1} g_{2} g_{3} g_{2} g_{1}^{-1} g_{2}^{-1} g_{3}^{-1} g_{2}^{-1} g_{1}^{-1}- \\
g_{3}^{-1} g_{1}=1\end{array}$ \\
\hline & \multicolumn{2}{|c|}{$\Gamma_{-7}(12,18)^{a b} \cong \mathbb{Z}^{3}, N=12$} \\
\hline \multirow[t]{2}{*}{$\Gamma_{-7}(12,19)$} & $\begin{array}{l}g_{1}=A C^{-1} \\
g_{2}=A^{3} \\
g_{3}=B A C^{-1} B \\
g_{4}=B A^{3} B\end{array}$ & $\begin{array}{l}\text { (1) } g_{1} g_{2} g_{1}^{-1} g_{2}^{-1}=1 \\
\text { (2) } g_{3} g_{4} g_{3}^{-1} g_{4}^{-1}=1 \\
\text { (3) } g_{1}^{2} g_{2}^{-1} g_{3}^{-1} g_{1} g_{3}^{-1} g_{4} g_{1} g_{3}^{-1} g_{1} g_{2}^{-1} g_{3}^{-1} g_{2} g_{3}^{-2} g_{4}- \\
g_{1} g_{3}^{-1} g_{2} g_{2}^{-1} g_{3}^{-1} g_{1} g_{3}^{-1} g_{4} g_{1} g_{3}^{-1}=1\end{array}$ \\
\hline & \multicolumn{2}{|c|}{$\Gamma_{-7}(12,19)^{a b}=\mathbb{Z}^{3}, N=6$} \\
\hline \multirow[t]{2}{*}{$\Gamma_{-7}(12,20)$} & $\begin{array}{l}g_{1}=C \\
g_{2}=A^{4} B \\
g_{3}=A^{2} B A^{2}\end{array}$ & $\begin{array}{l}\text { (1) } g_{1} g_{2} g_{1}^{-1} g_{2}^{-1} g_{1} g_{3} g_{1}^{-1} g_{3}^{-1} g_{1} g_{3} g_{1}^{-1} g_{2}^{-1}=1 \\
\text { (2) } g_{1}^{2} g_{3} g_{1}^{-1} g_{3}^{-1} g_{1} g_{2}^{-1} g_{1}^{-1} g_{2} g_{1} g_{2}^{-1} g_{1}^{-1} g_{2} g_{1}^{-1} g_{2}^{-1} g_{1}- \\
g_{2} g_{1}^{-1} g_{3} g_{2}^{-1} g_{1} g_{2} g_{1}^{-1} g_{2}^{-1}=1\end{array}$ \\
\hline & \multicolumn{2}{|c|}{$\Gamma_{-7}(12,20)^{a b} \cong \mathbb{Z}^{2}, N=3$} \\
\hline \multirow[t]{2}{*}{$\Gamma_{-7}(12,21)$} & $\begin{array}{l}g_{1}=A \\
g_{2}=B A^{2} C^{-2} B \\
g_{3}=B C^{-1} B C^{-1}\end{array}$ & $\begin{array}{l}\text { (1) } g_{1} g_{2} g_{1}^{-1} g_{2}^{-1} g_{3} g_{2}^{-1} g_{1}^{-1} g_{3} g_{1} g_{3}^{-1} g_{1} g_{3} g_{1}^{-1} g_{3}^{-1}=1 \\
\text { (2) } g_{1} g_{2} g_{1}^{-1} g_{2}^{-1} g_{3} g_{2}^{-1} g_{3} g_{1}^{-1} g_{3}^{-1} g_{2}^{-1} g_{3} g_{1} g_{3}^{-1} g_{2} g_{3}^{-1}- \\
g_{2}=1\end{array}$ \\
\hline & \multicolumn{2}{|c|}{$\Gamma_{-7}(12,21)^{a b} \cong \mathbb{Z}^{2}, N=3$} \\
\hline \multirow[t]{2}{*}{$\Gamma_{-7}(12,22)$} & $\begin{array}{l}g_{1}=A \\
g_{2}=B C^{-1} B C^{-1} \\
g_{3}=B A^{-1} C B C\end{array}$ & $\begin{array}{l}\text { (1) } g_{1} g_{2} g_{3} g_{1}^{-1} g_{3}^{-1} g_{1} g_{3} g_{1}^{-1} g_{2} g_{1}^{-1} g_{2}^{-1}=1 \\
\text { (2) } g_{1} g_{2} g_{1} g_{3} g_{1}^{-1} g_{3}^{-1} g_{2} g_{1} g_{2}^{-1} g_{3}^{-1} g_{1}^{-1} g_{3} g_{1} g_{3}^{-1} g_{1}^{-1}- \\
g_{2}^{-1} g_{3} g_{2} g_{1}^{-1} g_{2}^{-1}=1\end{array}$ \\
\hline & \multicolumn{2}{|c|}{$\Gamma_{-7}(12,22)^{a b} \cong \mathbb{Z}^{2}, N=6$} \\
\hline
\end{tabular}


TABLE 3 (continued)

\begin{tabular}{|c|c|c|}
\hline$\Gamma_{-7}(12,23)$ & $\begin{array}{l}g_{1}=A \\
g_{2}=B C^{2} B \\
g_{3}=B A C B C^{-1}\end{array}$ & $\begin{array}{l}\text { (1) } g_{1} g_{3} g_{1} g_{3} g_{1} g_{3}^{-1} g_{2} g_{3} g_{1}^{-1} g_{3}^{-1} g_{1}^{-1} g_{3}^{-1} g_{1}^{-1} g_{2}^{-1}=1 \\
\text { (2) } g_{1} g_{2} g_{1} g_{2}^{-1} g_{3} g_{1}^{-1} g_{3}^{-1} g_{2} g_{1}^{-1} g_{2}^{-1} g_{3} g_{1} g_{3}^{-1} g_{2} g_{1}^{-1}- \\
g_{2}^{-1} g_{1} g_{3}=1\end{array}$ \\
\hline & \multicolumn{2}{|c|}{$\Gamma_{-7}(12,23)^{a b} \cong \mathbb{Z}^{2}, N=3$} \\
\hline \multirow[t]{2}{*}{$\Gamma_{-7}(12,24)$} & $\begin{array}{l}g_{1}=A C^{-1} \\
g_{2}=B C^{4} \\
g_{3}=A C^{3} B\end{array}$ & $\begin{array}{l}\text { (1) } g_{1} g_{2} g_{1} g_{3}^{-1} g_{1} g_{3} g_{1}^{-1} g_{3}^{-1} g_{1}^{-1} g_{2} g_{1}^{-1} g_{2}^{-1}=1 \\
\text { (2) } g_{1}^{2} g_{3} g_{1} g_{2} g_{1} g_{2}^{-1} g_{1}^{-1} g_{2} g_{1}^{-1} g_{2}^{-1} g_{1} g_{2} g_{1}^{-1} g_{2}^{-1} g_{1}^{-1}- \\
g_{3}^{-1} g_{1}^{-1} g_{3} g_{2}^{-1} g_{1}^{-1} g_{2} g_{1} g_{2}^{-1}=1 \\
\end{array}$ \\
\hline & \multicolumn{2}{|c|}{$\Gamma_{-7}(12,24)^{a b} \cong \mathbb{Z}^{2}, N=3$} \\
\hline \multirow[t]{2}{*}{$\Gamma_{-7}(12,25)$} & $\begin{array}{l}g_{1}=B A^{-1} \\
g_{2}=C^{2} \\
g_{3}=A C B C^{2} B C^{-1} \\
g_{4}=C B C^{2} B C^{-1} A^{-1}\end{array}$ & $\begin{array}{l}\text { (1) } g_{1}^{3}=1 \\
\text { (2) } g_{1} g_{2}^{-1} g_{3} g_{4} g_{2} g_{1}^{-1} g_{2}^{-1} g_{3} g_{1}^{-1} g_{3}^{-1} g_{4}^{-1} g_{1} g_{3}^{-1} g_{2}=1 \\
\text { (3) }\left(g_{1} g_{2}^{-1} g_{3}^{2} g_{1} g_{3}^{-1} g_{2} g_{1} g_{2}^{-1} g_{3}^{-1} g_{2}\right)^{3}=1 \\
\text { (4) } g_{1} g_{2} g_{1}^{-1} g_{2}^{-1} g_{3} g_{1}^{-1} g_{3}^{-2} g_{2} g_{1}^{-1} g_{2}^{-2} g_{3} g_{1}^{-1} g_{2}^{-1} g_{1} g_{3}^{-1}- \\
g_{2} g_{1} g_{2}^{-1} g_{3}^{2} g_{1} g_{3}^{-1} g_{2}=1 \\
\text { (5) } g_{1} g_{2}^{-1} g_{3} g_{1} g_{3}^{-1} g_{2} g_{1} g_{2}^{-1} g_{3}^{2} g_{1} g_{3}^{-2} g_{2} g_{1}^{-1} g_{2}^{-1} g_{3} g_{1}^{-1}- \\
g_{4} g_{3}^{-1} g_{2} g_{1} g_{2}^{-1} g_{3} g_{4}^{-1} g_{1} g_{3}^{-1} g_{2}=1\end{array}$ \\
\hline & \multicolumn{2}{|c|}{$\Gamma_{-7}(12,25)^{a b} \cong \mathbb{Z}^{3}$, not torsion-free } \\
\hline \multirow[t]{2}{*}{$\Gamma_{-7}(12,26)$} & $\begin{array}{l}g_{1}=B A^{-1} \\
g_{2}=A C^{2} \\
g_{3}=A^{2} \\
g_{4}=C B A C^{2} B C \\
g_{5}=C^{-1} B C^{2} B C^{-1}\end{array}$ & $\begin{array}{l}\text { (1) } g_{1}^{3}=1 \\
\text { (2) } g_{2} g_{3} g_{2}^{-1} g_{3}^{-1}=1 \\
\text { (3) }\left(g_{1} g_{3}\right)^{3}=1 \\
\text { (4) } g_{1} g_{3} g_{5} g_{1}^{-1} g_{4} g_{5} g_{1} g_{5}^{-1} g_{3}^{-1} g_{1}^{-1} g_{4}^{-1} g_{5}^{-1}=1 \\
\text { (5) } g_{1} g_{3} g_{5} g_{1}^{-1} g_{2} g_{3}^{-1} g_{1} g_{3} g_{5} g_{1}^{-1} g_{5}^{-1} g_{2}^{-1} g_{5}^{-1}=1 \\
\text { (6) } g_{1} g_{2} g_{4}^{-1} g_{1} g_{5}^{-1} g_{3}^{-1} g_{1}^{-1} g_{5} g_{2} g_{3}^{-1} g_{1} g_{3} g_{2}^{-1} g_{5}^{-1} g_{1}- \\
g_{3} g_{5} g_{1}^{-1} g_{4} g_{3} g_{2}^{-1}=1\end{array}$ \\
\hline & \multicolumn{2}{|c|}{$\Gamma_{-7}(12,26)^{a b} \cong \mathbb{Z}^{3}$, not torsion-free } \\
\hline \multirow[t]{2}{*}{$\Gamma_{-7}(12,27)$} & $\begin{array}{l}g_{1}=B A^{-1} \\
g_{2}=C B C \\
g_{3^{\prime}}=A C B C^{-3} \\
g_{4}=A^{-1} C^{6}\end{array}$ & $\begin{array}{l}\text { (1) } g_{1}^{3}=1 \\
\text { (2) } g_{1} g_{2} g_{4}^{-1} g_{3}^{-2} g_{1}^{-1} g_{4} g_{1} g_{3}^{2} g_{4} g_{2}^{-1} g_{1}^{-1} g_{2} g_{4}^{-1} g_{3}^{-2} g_{4}- \\
g_{3}^{2} g_{4} g_{2}^{-1}=1 \\
\text { (3) } g_{1} g_{2} g_{4}^{-1} g_{3} g_{4} g_{2}^{-1} g_{1} g_{2} g_{4}^{-1} g_{3}^{-2} g_{1}^{-1} g_{2} g_{1} g_{3}^{2} g_{4} g_{2}^{-1}- \\
g_{1} g_{2} g_{4}^{-1} g_{3}^{-2} g_{1}^{-1} g_{2}^{-1} g_{3} g_{4} g_{2}^{-1}=1 \\
\text { (4) } g_{1} g_{2} g_{4}^{-1} g_{3}^{-2} g_{1}^{-1} g_{2} g_{3}^{2} g_{4} g_{2}^{-1} g_{1} g_{2} g_{4}^{-1} g_{3}^{-2} g_{1}^{-1} g_{2}^{-1}- \\
g_{3}^{3} g_{4} g_{2}^{-1} g_{1} g_{2} g_{4}^{-1} g_{3}^{-2} g_{1}^{-1} g_{3} g_{4} g_{2}^{-1}=1\end{array}$ \\
\hline & \multicolumn{2}{|c|}{$\Gamma_{-7}(12,27)^{a b} \cong \mathbb{Z}^{3}$, not torsion-free } \\
\hline
\end{tabular}




\section{TABLE 4}

All subgroups of index $\leq 12$ in $\Gamma_{-1}$ with torsion-free commutator factor-group, up to $\Gamma_{-1}$ conjugacy

\begin{tabular}{|c|c|c|}
\hline & Generators & Relations \\
\hline \multirow[t]{2}{*}{$\Gamma_{-1}(12,1)$} & $\begin{array}{l}g_{1}=C \\
g_{2}=B C A^{-1} B\end{array}$ & $\begin{array}{l}\text { (1) } g_{1} g_{2} g_{1}^{-1} g_{2} g_{1} g_{2}^{-1} g_{1} g_{2} g_{1}^{-1} g_{2}^{-1} g_{1} g_{2}^{-1} g_{1}^{-1} g_{2} g_{1}^{-1}- \\
g_{2}^{-1}=1\end{array}$ \\
\hline & \multicolumn{2}{|c|}{$\Gamma_{-1}(12,1) \cong \mathbb{Z}^{2}$, torsion-free, link complement group $(4.2)$} \\
\hline \multirow[t]{2}{*}{$\Gamma_{-1}(12,2)$} & $\begin{array}{l}g_{1}=C \\
g_{2}=B C A B\end{array}$ & $\begin{array}{l}\text { (1) } g_{1} g_{2} g_{1}^{-1} g_{2} g_{1} g_{2}^{-1} g_{1} g_{2} g_{1}^{-1} g_{2}^{-1} g_{1} g_{2}^{-1} g_{1}^{-1} g_{2} g_{1}^{-1}- \\
g_{2}^{-1}=1\end{array}$ \\
\hline & \multicolumn{2}{|c|}{ Iso $(H)$-conjugate to $\Gamma_{-1}(12,1)$} \\
\hline \multirow[t]{2}{*}{$\Gamma_{-1}(12,3)$} & $\begin{array}{l}g_{1}=A \\
g_{2}=B C A^{-1} B\end{array}$ & $\begin{array}{l}\text { (1) } g_{1} g_{2} g_{1}^{-1} g_{2} g_{1} g_{2}^{-1} g_{1} g_{2} g_{1}^{-1} g_{2}^{-1} g_{1} g_{2}^{-1} g_{1}^{-1} g_{2} g_{1}^{-1}- \\
g_{2}^{-1}=1\end{array}$ \\
\hline & \multicolumn{2}{|c|}{ Iso $(H)$-conjugate to $\Gamma_{-1}(12,1)$} \\
\hline \multirow[t]{2}{*}{$\Gamma_{-1}(12,4)$} & $\begin{array}{l}g_{1}=A \\
g_{2}=B C A B\end{array}$ & $\begin{array}{l}\text { (1) } g_{1} g_{2} g_{1} g_{2}^{-1} g_{1}^{-1} g_{2} g_{1} g_{2} g_{1}^{-1} g_{2}^{-1} g_{1}^{-1} g_{2} g_{1} g_{2}^{-1} g_{1}^{-1}- \\
g_{2}^{-1}=1\end{array}$ \\
\hline & \multicolumn{2}{|c|}{ Iso $(H)$-conjugate to $\Gamma_{-1}(12,1)$} \\
\hline \multirow[t]{2}{*}{$\Gamma_{-1}(12,5)$} & $\begin{array}{l}g_{1}=A \\
g_{2}=C \\
g_{3}=B A C^{2} B\end{array}$ & $\begin{array}{l}\text { (1) } g_{1} g_{2} g_{1}^{-1} g_{2}^{-1}=1 \\
\text { (2) } g_{1} g_{2}^{-1} g_{3} g_{2}^{-1} g_{1} g_{3} g_{2}^{-1} g_{3} g_{1} g_{3} g_{2}^{-1} g_{3}=1 \\
\text { (3) } g_{1} g_{3} g_{1}^{-1} g_{3}^{-1} g_{2} g_{3}^{-1} g_{1}^{-1} g_{3}^{-1} g_{1} g_{3} g_{2}^{-1} g_{3}=1\end{array}$ \\
\hline & \multicolumn{2}{|c|}{$\Gamma_{-1}(12,5)^{a b} \cong \mathbb{Z}^{2}$, link complement group (4.2) } \\
\hline \multirow[t]{2}{*}{$\Gamma_{-1}(12,6)$} & $\begin{array}{l}g_{1}=A \\
g_{2}=C^{-1} \\
g_{3}=B A C^{-2} B\end{array}$ & $\begin{array}{l}\text { (1) } g_{1} g_{2} g_{1}^{-1} g_{2}^{-1}=1 \\
\text { (2) } g_{1} g_{2}^{-1} g_{3} g_{2}^{-1} g_{1} g_{3} g_{2}^{-1} g_{3} g_{1} g_{3} g_{2}^{-1} g_{3}=1 \\
\text { (3) } g_{1} g_{3} g_{1}^{-1} g_{3}^{-1} g_{2} g_{3}^{-1} g_{1}^{-1} g_{1} g_{3} g_{2}^{-1} g_{3}=1\end{array}$ \\
\hline & \multicolumn{2}{|c|}{ Iso $(H)$-conjugate to $\Gamma_{-1}(12,5)$} \\
\hline
\end{tabular}


TABLE 5

All subgroups of index $\leq 12$ in $\Gamma_{-2}$ with torsionfree commutator factor group, up to $\Gamma_{-2}$ conjugacy

\begin{tabular}{|c|c|c|}
\hline & Generators & Relations \\
\hline \multirow[t]{2}{*}{$\Gamma_{-2}(12,1)$} & $\begin{array}{l}g_{1}=B C^{-1} \\
g_{2}=A^{2} C \\
g_{3}=A^{-1} B C B C\end{array}$ & $\begin{array}{l}\text { (1) } g_{1}^{2} g_{2} g_{3} g_{2}^{-1} g_{3}^{-1} g_{1} g_{2} g_{3} g_{2}^{-1} g_{1}^{-1} g_{3}^{-1} g_{2}^{-1} g_{1}^{-1} g_{3}=1 \\
\text { (2) } g_{1} g_{2} g_{1}^{-1} g_{2} g_{3}^{-1} g_{2}^{-1} g_{1}^{-1} g_{3} g_{2} g_{3}^{-1} g_{1}^{-1} g_{2} g_{3}^{-1} g_{2}^{-1} g_{1}^{-1}- \\
g_{3} g_{2}^{-1} g_{3}^{-1} g_{1} g_{2} g_{3} g_{2}^{-1} g_{1} g_{3} g_{2}^{-1} g_{3}^{-1} g_{1} g_{2} g_{3} g_{2}^{-1}=1\end{array}$ \\
\hline & \multicolumn{2}{|c|}{$\Gamma_{-7}(12,1)^{a b}=\mathbb{Z}^{2}$, torsion-free, link complement group $(5.2)$} \\
\hline \multirow[t]{2}{*}{$\Gamma_{-2}(12,2)$} & $\begin{array}{l}g_{1}=B C^{-1} \\
g_{2}=A^{2} C^{-1} \\
g_{3}=A B C B C\end{array}$ & $\begin{array}{l}\text { (1) } g_{1}^{2} g_{2} g_{3} g_{2}^{-1} g_{3}^{-1} g_{1} g_{2} g_{3} g_{2}^{-1} g_{1}^{-1} g_{3}^{-1} g_{2}^{-1} g_{1}^{-1} g_{3}=1 \\
\text { (2) } g_{1} g_{2} g_{1}^{-1} g_{2} g_{3}^{-1} g_{2}^{-1} g_{1}^{-1} g_{3} g_{2} g_{3}^{-1} g_{1}^{-1} g_{2} g_{3}^{-1} g_{2}^{-1} g_{1}^{-1}- \\
g_{3} g_{2}^{-1} g_{3}^{-1} g_{1} g_{2} g_{3} g_{2}^{-1} g_{1} g_{3} g_{2}^{-1} g_{3}^{-1} g_{1} g_{2} g_{3} g_{2}^{-1}=1\end{array}$ \\
\hline & \multicolumn{2}{|c|}{ Iso $(H)$-conjugate to $\Gamma_{-2}(12,1)$} \\
\hline \multirow[t]{2}{*}{$\Gamma_{-2}(12,3)$} & $\begin{array}{l}g_{1}=A \\
g_{2}=B A C^{-1} B \\
g_{3}=B C^{3} B C\end{array}$ & $\begin{array}{l}\text { (1) } g_{1} g_{2}^{2} g_{3} g_{1} g_{3}^{-1} g_{2} g_{3} g_{2}=1 \\
\text { (2) } g_{1} g_{2} g_{1} g_{2}^{-1} g_{1}^{-1} g_{2} g_{1} g_{2} g_{1}^{-1} g_{2}^{-1} g_{1}^{-1} g_{3}^{-1} g_{2}^{-3} g_{1}^{-1}- \\
g_{2}^{-1} g_{1} g_{2} g_{1} g_{2}^{-1} g_{1}^{-1} g_{2} g_{1} g_{2}^{3} g_{3} g_{1} g_{2} g_{1} g_{2}^{-1} g_{1}^{-1} g_{2}^{-1}- \\
g_{1} g_{2} g_{1}^{-1} g_{2}^{-1} g_{1}^{-1} g_{2} g_{1} g_{2}^{3} g_{3}=1\end{array}$ \\
\hline & \multicolumn{2}{|c|}{$\Gamma_{-2}(12,3)^{a b}=\mathbb{Z}^{2}$, torsion-free, link complement group $(5.2)$} \\
\hline \multirow[t]{2}{*}{$\Gamma_{-2}(12,4)$} & $\begin{array}{l}g_{1}=A \\
g_{2}=B A C B \\
g_{3}=B C^{-3} B C^{-1}\end{array}$ & $\begin{array}{l}\text { (1) } g_{1} g_{2}^{2} g_{3} g_{1} g_{3}^{-1} g_{2} g_{3} g_{2}=1 \\
\text { (2) } g_{1} g_{2} g_{1} g_{2}^{-1} g_{1}^{-1} g_{2} g_{1} g_{2} g_{1}^{-1} g_{2}^{-1} g_{1}^{-1} g_{3}^{-1} g_{2}^{-3} g_{1}^{-1} g_{2}^{-1}- \\
g_{1} g_{2} g_{1} g_{2}^{-1} g_{1}^{-1} g_{2} g_{1} g_{2}^{3} g_{3} g_{1} g_{2} g_{1} g_{2}^{-1} g_{1}^{-1} g_{2}^{-1} g_{1}- \\
g_{2} g_{1}^{-1} g_{2}^{-1} g_{1}^{-1} g_{2} g_{1} g_{2}^{3} g_{3}=1\end{array}$ \\
\hline & \multicolumn{2}{|c|}{ Iso $(H)$-conjugate to $\Gamma_{-2}(12,3)$} \\
\hline \multirow[t]{2}{*}{$\Gamma_{-2}(12,5)$} & $\begin{array}{l}g_{1}=A^{-1} B A^{-1} B A \\
g_{2}=C^{2} A^{-1} \\
g_{3}=C A^{2}\end{array}$ & $\begin{array}{l}\text { (1) } g_{2} g_{3} g_{2}^{-1} g_{3}^{-1}=1 \\
\text { (2) } g_{1} g_{3}^{-1} g_{1} g_{3}^{-1} g_{2} g_{1} g_{3}^{-1} g_{1} g_{3} g_{1}^{-1} g_{2}^{-1} g_{3} g_{1}^{-1} g_{3} g_{1}^{-1}- \\
g_{3}^{-1} g_{1} g_{3}^{-1} g_{2} g_{1} g_{3}^{-1} g_{1} g_{2} g_{1} g_{3}^{-1} g_{1} g_{3}^{-1} g_{2} g_{1} g_{3}^{-1} g_{1}^{-1}- \\
g_{3} g_{1}^{-1} g_{2}^{-1} g_{3} g_{1}^{-1} g_{3} g_{1}^{-1} g_{3}^{-1} g_{1} g_{3}^{-1} g_{2} g_{1} g_{3}^{-1} g_{1} g_{3}- \\
g_{1}^{-1} g_{2}^{-1} g_{3} g_{1}^{-1} g_{3} g_{1}^{-1} g_{2}^{-1} g_{1}^{-1} g_{3} g_{1}^{-1} g_{2}^{-1} g_{3} g_{1}^{-1} g_{3}=1\end{array}$ \\
\hline & \multicolumn{2}{|c|}{$\Gamma_{-2}(12,5)^{a b} \cong \mathbb{Z}^{3}$, generated by unipotent elements } \\
\hline \multirow[t]{2}{*}{$\Gamma_{-2}(12,6)$} & $\begin{array}{l}g_{1}=A B A B A^{-1} \\
g_{2}=C^{2} A \\
g_{3}=C A^{-2}\end{array}$ & $\begin{array}{l}\text { (1) } g_{2} g_{3} g_{2}^{-1} g_{3}^{-1}=1 \\
\text { (2) } g_{1} g_{3}^{-1} g_{1} g_{3}^{-1} g_{2} g_{1} g_{3}^{-1} g_{1} g_{3} g_{1}^{-1} g_{2}^{-1} g_{3} g_{1}^{-1} g_{3} g_{1}^{-1} g_{3}^{-1}- \\
g_{1} g_{3}^{-1} g_{2} g_{1} g_{3}^{-1} g_{1} g_{2} g_{1} g_{3}^{-1} g_{1} g_{3}^{-1} g_{2} g_{1} g_{3}^{-1} g_{1}^{-1} g_{3} g_{1}^{-1}- \\
g_{2}^{-1} g_{3} g_{1}^{-1} g_{3} g_{1}^{-1} g_{3}^{-1} g_{1} g_{3}^{-1} g_{2} g_{1} g_{3}^{-1} g_{1} g_{3} g_{1}^{-1} g_{2}^{-1} g_{3}- \\
g_{1}^{-1} g_{3} g_{1}^{-1} g_{2}^{-1} g_{1}^{-1} g_{3} g_{1}^{-1} g_{2}^{-1} g_{3} g_{1}^{-1} g_{3}=1\end{array}$ \\
\hline & \multicolumn{2}{|c|}{ Iso $(H)$-conjugate to $\Gamma_{-2}(12,5)$} \\
\hline
\end{tabular}


TABLE 5 (continued)

\begin{tabular}{|c|c|c|}
\hline$\Gamma_{-2}(12,7)$ & $\begin{array}{l}g_{1}=B C^{-1} \\
g_{2}=A^{-1} C^{2} \\
g_{3}=A^{-2} B A C\end{array}$ & $\begin{array}{l}\text { (1) } g_{1}^{2} g_{2} g_{1}^{2} g_{2} g_{1}^{-1} g_{3}^{-1} g_{2} g_{3}^{-2} g_{1}^{-1} g_{2}^{-1} g_{1}^{-2} g_{2}^{-1} g_{3}^{2} g_{2}^{-1} g_{3}=1 \\
\text { (2) } g_{1}^{2} g_{2} g_{1} g_{3}^{2} g_{2}^{-1} g_{3} g_{1}^{-1} g_{2}^{-1} g_{1}^{-2} g_{3}^{-1} g_{2} g_{3}^{-2} g_{2} g_{3}^{-1} g_{2}^{-1}- \\
g_{3}=1\end{array}$ \\
\hline & \multicolumn{2}{|l|}{$\Gamma_{-2}(12,7) \cong \mathbb{Z}^{3}$} \\
\hline \multirow[t]{2}{*}{$\Gamma_{-2}(12,8)$} & $\begin{array}{l}g_{1}=B C^{-1} \\
g_{2}=A C^{2} \\
g_{3}=A^{2} B A^{-1} C\end{array}$ & $\begin{array}{l}\text { (1) } g_{1}^{2} g_{2} g_{1}^{2} g_{2} g_{1}^{-1} g_{3}^{-1} g_{2} g_{3}^{-2} g_{1}^{-1} g_{2}^{-1} g_{1}^{-2} g_{2}^{-1} g_{2}^{-1} g_{3}^{2} g_{2}^{-1} g_{3}=1 \\
\text { (2) } g_{1}^{2} g_{2} g_{1} g_{3}^{2} g_{2}^{-1} g_{3} g_{1}^{-1} g_{2}^{-1} g_{1}^{-2} g_{3}^{-1} g_{2} g_{3}^{-2} g_{2} g_{3}^{-1} g_{2}^{-1}- \\
g_{3}=1\end{array}$ \\
\hline & \multicolumn{2}{|c|}{ Iso $(H)$-conjugate to $\Gamma_{-2}(12,7)$} \\
\hline \multirow[t]{2}{*}{$\Gamma_{-2}(12,9)$} & $\begin{array}{l}g_{1}=A C^{-1} \\
g_{2}=C^{3} \\
g_{3}=B A C^{-1} B \\
g_{4}=A^{-1} B A^{-1} C B A\end{array}$ & $\begin{array}{l}\text { (1) } g_{1} g_{2} g_{1}^{-1} g_{2}^{-1}=1 \\
\text { (2) } g_{1} g_{2} g_{1} g_{4}^{-1} g_{3}^{-1} g_{4}^{-1} g_{3} g_{4} g_{1}^{-1} g_{2}^{-1} g_{1}^{-1} g_{3}^{-1} g_{1} g_{4} g_{1}^{-1}- \\
g_{3}=1 \\
\text { (3) } g_{1} g_{2} g_{1} g_{4}^{-1} g_{1}^{-1} g_{3} g_{1} g_{4} g_{1}^{-1} g_{2}^{-1} g_{1}^{-1} g_{3}^{-1} g_{4}^{-1} g_{3}^{-1}- \\
g_{4} g_{3}=1\end{array}$ \\
\hline & \multicolumn{2}{|c|}{$\Gamma_{-}(12,9)^{a b} \cong \mathbb{Z}^{4}$, generated by unipotent elements } \\
\hline \multirow[t]{2}{*}{$\Gamma_{-2}(12,10)$} & $\begin{array}{l}g_{1}=A C \\
g_{2}=C^{-3} \\
g_{3}=B A C B \\
g_{4}=A^{-1} B A^{-1} C^{-1} B A\end{array}$ & $\begin{array}{l}\text { (1) } g_{1} g_{2} g_{1}^{-1} g_{2}^{-1}=1 \\
\text { (2) } g_{1} g_{2} g_{1} g_{4}^{-1} g_{3}^{-1} g_{4}^{-1} g_{3} g_{4} g_{1}^{-1} g_{2}^{-1} g_{1}^{-1} g_{3}^{-1} g_{1} g_{4} g_{1}^{-1}- \\
g_{3}=1 \\
\text { (3) } g_{1} g_{2} g_{1} g_{4}^{-1} g_{1}^{-1} g_{3} g_{1} g_{4} g_{1}^{-1} g_{2}^{-1} g_{1}^{-1} g_{3}^{-1} g_{4}^{-1} g_{3}^{-1}- \\
g_{4} g_{3}=1\end{array}$ \\
\hline & \multicolumn{2}{|c|}{ Iso $(H)$-conjugate to $\Gamma_{-2}(12,9)$} \\
\hline$\Gamma_{-2}(12,11)$ & $\begin{array}{l}g_{1}=A \\
g_{2}=B C^{-1} B C^{-1} \\
g_{3}=C^{-1} B C^{-1} B \\
g_{4}=B C^{-2} A^{2} B\end{array}$ & $\begin{array}{l}\text { (1) }\left(g_{1} g_{2} g_{1}^{-1} g_{2}^{-1}\right)^{2}=1 \\
\text { (2) }\left(g_{1} g_{3} g_{1}^{-1} g_{3}^{-1}\right)^{2}=1 \\
\text { (3) } g_{1} g_{2} g_{1}^{-1} g_{2}^{-1} g_{3} g_{4}^{-1} g_{2} g_{1} g_{2}^{-1} g_{4} g_{3}^{-1}=1 \\
\text { (4) } g_{1} g_{2}^{-1} g_{4} g_{3}^{-1} g_{1} g_{3} g_{1}^{-1} g_{4}^{-1} g_{2} g_{3}^{-1} g_{1}^{-1} g_{3}=1 \\
\text { (5) } g_{1} g_{2}^{-1} g_{4} g_{3}^{-1} g_{1} g_{3} g_{4} g_{3}^{-1} g_{2} g_{1}^{-1} g_{2}^{-1} g_{3} g_{4}^{-1} g_{1}^{-1}- \\
g_{4}^{-1} g_{2}=1\end{array}$ \\
\hline & \multicolumn{2}{|c|}{$\Gamma_{-2}(12,11)^{a b} \cong \mathbb{Z}^{4}$, not torsion-free } \\
\hline
\end{tabular}


TABLE 6

All subgroups of index 12 in $\Gamma_{-3}$, up to $\Gamma_{-3}$ conjugacy

\begin{tabular}{|c|c|c|}
\hline & Generators & Relations \\
\hline \multirow[t]{2}{*}{$\overline{\Gamma_{-3}(12,1)}$} & $\begin{array}{l}g_{1}=A \\
g_{2}=B C B\end{array}$ & (1) $g_{1} g_{2} g_{1}^{-1} g_{2} g_{1} g_{2}^{-1} g_{1}^{-1} g_{2} g_{1}^{-1} g_{2}^{-1}=1$ \\
\hline & \multicolumn{2}{|c|}{$\Gamma_{-3}(12,1)^{a b} \cong \mathbb{Z}$, torsion-free, link complement group $(6.2), N=6$} \\
\hline \multirow[t]{2}{*}{$\Gamma_{-3}(12,2)$} & $\begin{array}{l}g_{1}=A^{2} \\
g_{2}=B C A B A C^{-1} B C^{-1} B\end{array}$ & (1) $g_{1} g_{2} g_{1} g_{2} g_{1} g_{2}^{2} g_{1} g_{2}^{-1} g_{1} g_{2}^{2}=1$ \\
\hline & \multicolumn{2}{|c|}{$\Gamma_{-3}(12,2)^{a b} \cong \mathbb{Z} \times \mathbb{Z} / 5 \mathbb{Z}$, torsion-free, $N=6$} \\
\hline \multirow[t]{2}{*}{$\Gamma_{-3}(12,3)$} & $\begin{array}{l}g_{1}=B A B C B A^{-1} \\
g_{2}=B A C^{2} B A^{-1}\end{array}$ & $\begin{array}{l}\text { (1) }\left(g_{1}^{2} g_{2}^{-1}\right)^{3}=1 \\
\text { (2) }\left(g_{1}^{3} g_{2}^{-1} g_{1} g_{2} g_{1} g_{2}^{-1}\right)^{3}=1 \\
\text { (3) } g_{1}^{3} g_{2}^{-1} g_{1} g_{2} g_{1} g_{2}^{-1} g_{1} g_{2} g_{1}^{-3} g_{2} g_{1}^{-1} g_{2}^{-1} g_{1} g_{2}^{-1} g_{1} g_{2}^{-1} g_{1}^{-1}- \\
g_{2} g_{1}^{-1} g_{2}^{-1} g_{1}^{-1} g_{2} g_{1}^{-1} g_{2} g_{1} g_{2}^{-1}=1\end{array}$ \\
\hline & \multicolumn{2}{|c|}{$\Gamma_{-3}(12,3)^{a b} \cong \mathbb{Z} / 3 \mathbb{Z} \times \mathbb{Z} / 9 \mathbb{Z}$, not torsion-free, $N=4$} \\
\hline$\Gamma_{-3}(12,4)$ & $\begin{array}{l}g_{1}=A B A C^{-1} B \\
g_{2}=B A B C B \\
g_{3}=C A^{-1}\end{array}$ & $\begin{array}{l}\text { (1) } g_{3} g_{1} g_{3}^{-1} g_{2} g_{3} g_{1}^{2} g_{2}^{-1}=1 \\
\text { (2) } g_{3} g_{1} g_{3}^{-1} g_{2}^{2} g_{1}^{-1} g_{3}^{-1} g_{2}=1 \\
\text { (3) } g_{3} g_{1}^{2} g_{2}^{-2} g_{3} g_{1} g_{2}^{-1}=1 \\
\text { (4) } g_{3} g_{1} g_{3} g_{1}^{-1} g_{2}^{-1} g_{3} g_{1}^{-3} g_{3}^{-1} g_{2} g_{3} g_{2}^{-2}=1 \\
\text { (5) } g_{3} g_{1} g_{3}^{-1} g_{2}^{-1} g_{1}^{-2} g_{3}^{-1} g_{2}^{-1} g_{1}^{-2} g_{3}^{-1} g_{2} g_{3}^{-1} g_{2}^{-2}=1 \\
\text { (6) }\left(g_{3} g_{1}^{2} g_{3} g_{2}^{-1}\right)^{3}=1 \\
\text { (7) } g_{3} g_{1}^{2} g_{3} g_{1}^{2} g_{3} g_{1}^{-2} g_{3}^{-1} g_{2} g_{3} g_{1}^{-2} g_{3}^{-1} g_{2}^{2}=1\end{array}$ \\
\hline
\end{tabular}

$\Gamma_{-3}(12,4)^{a b} \cong \mathbb{Z} / 3 \mathbb{Z} \times \mathbb{Z} / 9 \mathbb{Z}$, not torsion-free, $N=4$

\begin{tabular}{l|l|l}
\hline$\Gamma_{-3}(12,5)$ & $g_{1}=B A^{3} B$ & (1) $g_{3} g_{1}^{-1} g_{3}^{-1} g_{1}^{-1}=1$ \\
$g_{2}=C A^{-1}$ & (2) $g_{2} g_{3}^{-1} g_{1}^{-1} g_{2} g_{1}^{-1} g_{3}^{-1} g_{2} g_{1}^{-1} g_{3}^{-1}=1$ \\
$g_{3}=B C A^{-1} B$ & (3) $g_{2} g_{3}^{-1} g_{1}^{-1} g_{2} g_{4}^{-1} g_{2}^{-1} g_{4}^{-1} g_{2}^{-1} g_{3} g_{1} g_{3}^{-1} g_{1}^{-1}=1$ \\
$g_{4}=A B C A^{-1} B A^{-1}$ & (4) $\left(g_{2} g_{4} g_{1}^{-1} g_{3}^{-1}\right)^{3}=1$ \\
& (5) $g_{2} g_{3} g_{1} g_{4} g_{1}^{-1} g_{3}^{-1} g_{2} g_{4} g_{3} g_{2} g_{4} g_{1}^{-1} g_{3}^{-1}=1$ \\
& (6) $g_{2} g_{4} g_{3} g_{4}^{-1} g_{2}^{-1} g_{4} g_{1}^{-1} g_{3}^{-1} g_{4} g_{3} g_{4} g_{1}^{-1} g_{3}^{-1}=1$ \\
& (7) $g_{2} g_{4} g_{3}^{2} g_{1} g_{4}^{-1} g_{2}^{-1} g_{4} g_{1}^{-1} g_{3}^{-1} g_{4}^{2} g_{1}^{-1} g_{3}^{-1}=1$ \\
& (8) $g_{2}^{2} g_{4} g_{2}^{-1} g_{1} g_{3} g_{4} g_{1}^{-1} g_{3}^{-1} g_{2} g_{3} g_{2} g_{4} g_{1}^{-1} g_{3}^{-1}=1$ \\
& (9) $g_{2} g_{3} g_{1} g_{4}^{-1} g_{2} g_{3} g_{1} g_{4}^{-1} g_{2} g_{4} g_{1}^{-1} g_{3}^{-1} g_{4} g_{1}^{-1} g_{3}^{-1}=1$ \\
\hline
\end{tabular}


TABLE 6 (continued)

\begin{tabular}{|c|c|c|}
\hline \multirow[t]{2}{*}{$\Gamma_{-3}(12,6)$} & $\begin{array}{l}g_{1}=A^{4} \\
g_{2}=B \\
g_{3}=A^{-2} B A^{2} \\
g_{4}=C A^{-1}\end{array}$ & $\begin{array}{l}\text { (1) } g_{2}^{2}=1 \\
\text { (2) } g_{3}^{2}=1 \\
\text { (3) } g_{4} g_{1} g_{4}^{-1} g_{1}^{-1}=1 \\
\text { (4) } g_{2} g_{4}^{-2} g_{3} g_{4} g_{2}^{-1} g_{4}^{-2} g_{3}^{-1} g_{4}=1 \\
\text { (5) } g_{2} g_{4} g_{1} g_{3} g_{1}^{-1} g_{4}^{-1} g_{2}^{-1} g_{4} g_{2}^{-1} g_{4}^{-1} g_{3} g_{4}=1 \\
\text { (6) } g_{2} g_{4} g_{1} g_{3}^{-1} g_{4} g_{2} g_{4}^{-1} g_{3}^{-1} g_{4} g_{3}^{-1} g_{1}^{-1} g_{4}^{-1}=1 \\
\text { (7) } g_{2} g_{4}^{2} g_{1} g_{2} g_{4}^{-1} g_{3}^{-1} g_{4} g_{3}^{-1} g_{1}^{-1} g_{2}^{-1} g_{4}^{-1} g_{3} g_{4}=1 \\
\text { (8) }\left(g_{2} g_{4}^{2} g_{1} g_{3} g_{1}^{-1} g_{4}^{-1}\right)^{2}=1 \\
\text { (9) }\left(g_{2} g_{4}^{2} g_{1} g_{3}^{-1} g_{1}^{-1} g_{4}^{-1}\right)^{2}=1 \\
\text { (10) } g_{2} g_{4} g_{1} g_{3} g_{2}^{-1} g_{4} g_{1} g_{3}^{-1} g_{1}^{-1} g_{4}^{-1} g_{2}^{-1} g_{3}^{-1} g_{1}^{-1} a g_{4}^{-1}=1 \\
\text { (11) }\left(g_{2} g_{4} g_{1} g_{3} g_{1}^{-1} g_{4}^{-2}\right)^{2}=1 \\
\text { (12) } g_{2} g_{4}^{2} g_{1} g_{2} g_{4}^{-1} g_{2} g_{4}^{2} g_{1} g_{2} g_{4}^{-1} g_{3}^{-1} g_{1}^{-1} g_{2}^{-1} g_{4} g_{2}^{-1} g_{3}^{-1}- \\
g_{1}^{-1} g_{4}^{-1}=1\end{array}$ \\
\hline & \multicolumn{2}{|c|}{$\Gamma_{-3}(12,6)^{a b} \cong \mathbb{Z}$, not torsion-free, $N=6$} \\
\hline \multirow[t]{2}{*}{$\Gamma_{-3}(12,7)$} & $\begin{array}{l}g_{1}=A \\
g_{2}=B A^{3} B \\
g_{3}=B C B A^{-1} C^{-1} B\end{array}$ & $\begin{array}{l}\text { (1) } g_{3}^{2}=1 \\
\left.\text { (2) } g_{1} g_{2}\right)^{3}=1 \\
\text { (3) }\left(g_{1} g_{3}\right)^{3}=1 \\
\text { (4) }\left(g_{1} g_{3}^{-1}\right)^{3}=1 \\
\text { (5) } g_{1} g_{3} g_{1} g_{3}^{-1} g_{1}^{-1} g_{3} g_{1}^{-1} g_{3}^{-1}=1 \\
\text { (6) }\left(g_{1}^{2} g_{3}\right)^{3}=1 \\
\text { (7) }\left(g_{1}^{2} g_{3}^{-1}\right)^{3}=1 \\
\text { (8) } g_{1} g_{2} g_{1} g_{3}^{-1} g_{1} g_{2} g_{3} g_{1}^{-1} g_{3} g_{2} g_{1} g_{3}^{-1}=1 \\
\text { (9) } g_{1} g_{2}^{-1} g_{1} g_{3}^{-1} g_{1} g_{2}^{-1} g_{3} g_{1}^{-1} g_{3} g_{2}^{-1} g_{1} g_{3}^{-1}=1 \\
\text { (10) } g_{1} g_{2} g_{1}^{-1} g_{3} g_{1}^{-1} g_{2} g_{1} g_{3}^{-1} g_{1} g_{2}^{-1} g_{1}^{-1} g_{3} g_{1}^{-1} g_{2}^{-1} g_{1}- \\
g_{3}^{-1}=1\end{array}$ \\
\hline & \multicolumn{2}{|c|}{$\Gamma_{-3}(12,7)^{a b} \cong \mathbb{Z} / 3 \mathbb{Z} \times \mathbb{Z} / 3 \mathbb{Z} \times \mathbb{Z} / 3 \mathbb{Z}$, not torsion-free, $N=4$} \\
\hline
\end{tabular}




\section{REFERENCES}

1. P. Bauer, Linkaussenräume und 2-dimensionaler Deformationsretrakt von arithmetischen Quotienten $\Gamma \backslash H$, Diplomarbeit, Bonn, 1987.

2. L. Bianchi, Geometrische Darstellung der Gruppen linearer Substitutionen mit ganzen complexen Coeffizienten nebst Anwendungen auf die Zahlentheorie, Math. Ann. 38 (1891), 313333.

3. __ Sui gruppi di sostituzioni con coefficienti appartenenti a corpi quadratici imaginari, Math. Ann. 40 (1892), 332-412.

4. A. Brunner, M. Frame, Y. Lee and N. Wielenberg, Classifying torsion-free subgroups of the Picard group, Trans. Amer. Math. Soc. 282 (1984), 205-235.

5. A. Brunner, Y. Lee and N. Wielenberg, Polyhedral groups and graph amalgamation products, Topology Appl. 20 (1985), 289-304.

6. L. Clozel, On the cuspidal cohomology of arithmetic subgroups of $\mathrm{SL}(2 n)$ and the first Betti number of arithmetic 3-manifolds, Duke Math. J. 55 (1987), 475-486.

7. H. Coxeter and W. Moser, Generators and relations for discrete groups, Ergeb. Math. Grenzgeb., Neue Folge, Bd. 14, Springer, Berlin, Heidelberg, New York, 1965.

8. J. E. Cremona, Hyperbolic tessellations, modular symbols, and elliptic curves over complex quadratic fields, Compositio Math. 51 (1984), 275-323.

9. J. Elstrodt, F. Grunewald and J. Mennicke, PSL(2) over imaginary quadratic integers, Astérisque 94 (1982), 43-60.

10. B. Fine and M. Newman, The normal subgroup structure of the Picard group, Trans. Amer. Math. Soc. 302 (1987), 769-786.

11. D. Flöge, Zur Struktur der $\mathrm{PSL}_{2}$ über einigen imaginar quadratischen Zahlringen, Math. Z. 183 (1983), 255-279.

12. R. Fox, Free differential calculus. II, Ann. of Math. (2) 59 (1954), 196-210.

13. F. Grunewald and J. Mennicke, $\mathrm{SL}_{2}(O)$ and elliptic curves, Manuscript, Bielefeld, 1978.

14. F. Grunewald, $\mathrm{H}$. Helling and J. Mennicke, $\mathrm{SL}_{2}$ over complex quadratic number fields. I, Algebra i Logika 17 (1978), 512-580.

15. F. Grunewald and J. Schwermer, Free non-abelian quotients of $\mathrm{SL}_{2}$ over orders of imaginary quadratic number fields, J. Algebra 69 (1981), 294-304.

16. __ Arithmetic quotients of hyperbolic 3-space, cusp forms and link complements, Duke Math. J. 48 (1981), 351-358.

17. __ A non-vanishing theorem for the cuspidal cohomology of $\mathrm{SL}_{2}$ over imaginary quadratic integers, Math. Ann. 258 (1981), 183-200.

18. G. Harder, Period integrals of Eisenstein cohomology classes and special values of some $L$ functions, Number Theory Related to Fermat's Last Theorem, (N. Koblitz, ed.), Progress in Math., vol. 26, Birkhäuser, Stuttgart and Basel, 1982, pp. 103-142.

19. A. Hatcher, Hyperbolic structures of arithmetic type on some link complements, J. London Math. Soc. (2) 27 (1983), 345-355.

20. G. Havas, A Reidemeister-Schreier Program, Proceedings of the Second International Conference on Group Theory, Lecture Notes in Math., vol. 372, Springer-Verlag, Berlin, Heidelberg, New York, pp. 347-356.

21. G. Havas and S. Sterling, Integer matrices and abelian groups, EUROSAM '79, pp. 431451, International Symposium on Symbolic and Algebraic Manipulation, Marseille (1975), Lecture Notes in Computer Science, no. 72, Springer-Verlag, Berlin, Heidelberg, New York.

22. G. Havas, P. E. Kenne, J. S. Richardson and E. F. Robertson, A Tietze transformation program, Computational Group Theory, (M. D. Atkinson, ed.), Academic Press, New York, 1984.

23. P. Hilton and U. Stammbach, A course in homological algebra, Graduate Texts in Math. no. 4, Springer-Verlag, Berlin, Heidelberg, New York, 1978.

24. N. Krámer, Beiträge zur Arithmetik imaginär quadratischer Zahlkörper, Bonner Math. Schriften, vol. 191, Bonn, 1985. 
25. W. Magnus, A. Karrass and D. Solitar, Combinatorial group theory, Wiley, New York, London, Sydney, 1966.

26. E. Mendoza, Cohomology of $P G L_{2}$ over imaginary quadratic integers, Bonner Math. Schriften, vol. 128, Bonn, 1980.

27. G. Mostow, Strong rigidity of locally symmetric spaces, Ann. of Math. Studies, no. 78, Princeton Univ. Press, Princeton, N.J., 1973.

28. J. Neubüser, An elementary introduction to coset table methods in computational group theory, Groups St. Andrews 1981 (C. M. Campbell and E. F. Robertson, eds.), LMS Lecture Notes, vol. 71, Cambridge Univ. Press, London and New York.

29. E. Picard, Sur une groupe des transformations des points l'espace situés du meme coté d'un plan, Bull. Soc. Math. France 12 (1894), 43-47.

30. H. Poincaré, Mémoire sur les groupes kleinéens, Acta Math. 3 (1883), 49-92 (= Oeuvres, vol. 2, pp. 258-299).

31. G. Prasad, Strong rigidity of $\mathbb{Q}$-rank 1 lattices, Invent. Math. 21 (1973), 255-286.

32. R. Riley, A quadratic parabolic group, Math. Proc. Cambridge Philos. Soc. 77 (1975), 281288.

33. _ Applications of a computer implementation of Poincaré's theorem on fundamental polyhedra, Math. Comp. 40 (1983), 607-632.

34. J. Rohlfs, On the cuspidal cohomology of the Bianchi modular groups, Math. Z. 186 (1985), 253-269.

35. D. Rolfsen, Knots and links, Publish or Perish, Berkeley, Calif., 1976.

36. J. Schwermer, A note on link complements and arithmetic groups, Math. Ann. 249 (1980), 107-110.

37. J. Schwermer and K. Vogtmann, The integral homology of $\mathrm{SL}_{2}$ and $\mathrm{PSL}_{2}$ of Euclidean imaginary quadratic integers, Comment. Math. Helv. 58 (1983), 573-598.

38. J. P. Serre, Le problème des groupes de congruence pour $\mathrm{SL}_{2}$, Ann. of Math. (2) 92 (1972), 489-527.

39. T. A. Springer, Invariant theory, Lecture Notes in Math., vol. 585, Springer-Verlag, Berlin, Heidelberg, New York, 1977.

40. R. Swan, Generators and relations for certain special linear groups, Adv. in Math. 6 (1971), 1-78.

41. W. P. Thurston, The geometry and topology of 3-manifolds, Lecture Notes, Princeton Univ. Press, Princeton, N.J., 1978.

42. _ Three dimensional manifolds, Kleinian groups and hyperbolic geometry, Bull. Amer. Math. Soc. (N.S.) 6 (1982), 357-381.

43. K. Vogtmann, Rational homology of Bianchi groups, Math. Ann. 272 (1985), 399-419.

44. N. Wielenberg, The structure of certain subgroups of the Picard group, Math. Proc. Cambridge Philos. Soc. 84 (1978), 427-436.

45. __ Polyhedral orbifold groups, Low-Dimensional Topology and Kleinian Groups, (D. B. A. Epstein, ed.), London Math. Soc. 112, Cambridge Univ. Press, Cambridge, 1986.

Mathematisches Institut der Universität BonN, Wegelerstrasse 10, D-5300 BonN 1, West Germany

Mathematisch-Geographische Fakultät, Katholische Universität Eichstätt, OstenSTRASSE 26-28, D-8078 EICHSTÄTt, WEST GERMANY 